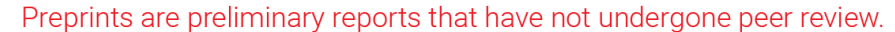 They should not be considered conclusive, used to inform clinical practice, or referenced by the media as validated information. \\ Reconfiguration of Multistable 3D Ferromagnetic Mesostructures Guided by Energy Landscape Surveys
}

Yi Li

University of Connecticut

\section{Sam Avis}

Durham University https://orcid.org/0000-0001-9637-9489

Junbo Chen

Syracuse University

Guangfu Wu

University of Connecticut

Teng Zhang ( $\nabla$ tzhang48@syr.edu )

Syracuse University https://orcid.org/0000-0001-5001-8485

Halim Kusumaatmaja ( $\square$ halim.kusumaatmaja@durham.ac.uk)

Durham University https://orcid.org/0000-0002-3392-9479

XUEJU WANG ( $\nabla$ xueju.wang@uconn.edu )

University of Connecticut https://orcid.org/0000-0002-0669-8759

\section{Article}

Keywords: Reconfigurable 3D structures, Magnetic actuation, Multistability, Energy landscape

Posted Date: September 15th, 2020

DOl: https://doi.org/10.21203/rs.3.rs-72622/v1

License: (9) (i) This work is licensed under a Creative Commons Attribution 4.0 International License.

Read Full License

Version of Record: A version of this preprint was published at Extreme Mechanics Letters on October 1st, 2021. See the published version at https://doi.org/10.1016/j.eml.2021.101428. 


\section{Reconfiguration of Multistable 3D Ferromagnetic Mesostructures Guided by Energy Landscape Surveys}

Yi Li ${ }^{a l}$, Sam Avis ${ }^{b l}$, Junbo Chen ${ }^{c}$, Guangfu Wu ${ }^{a}$, Teng Zhang ${ }^{c, d} *$, Halim Kusumaatmaja ${ }^{b}$, Хиејu Wang ${ }^{a, e *}$

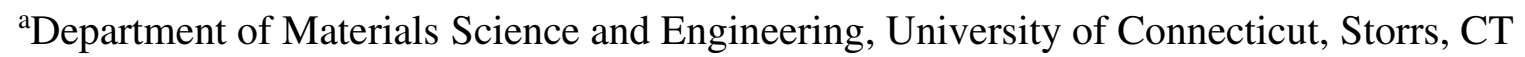
06250, USA

${ }^{\text {b} D e p a r t m e n t ~ o f ~ P h y s i c s, ~ D u r h a m ~ U n i v e r s i t y, ~ D u r h a m, ~ D H 1 ~ 3 L E, ~ E n g l a n d ~}$

${ }^{\mathrm{c}}$ Department of Mechanical and Aerospace Engineering, Syracuse University, Syracuse, NY, 13244, USA

${ }^{\mathrm{d} B i o I n s p i r e d ~ S y r a c u s e, ~ S y r a c u s e ~ U n i v e r s i t y, ~ S y r a c u s e, ~ N Y ~ 13244, ~ U S A ~}$

e Institute of Materials Science, University of Connecticut, Storrs, CT 06269, USA

${ }^{1}$ These authors contributed equally to this work.

*Corresponding authors. Email: xueju.wang@uconn.edu;

halim.kusumaatmaja@durham.ac.uk; tzhang48@syr.edu

Keywords: Reconfigurable 3D structures, Magnetic actuation, Multistability, Energy landscape 


\begin{abstract}
Reconfigurable three-dimensional (3D) structures that can reversibly change their geometries and thereby their functionalities are promising for a wide range of applications. Despite intensive studies, the lack of fundamental understanding of the highly nonlinear multistable states existing in these structures has significantly hindered the development of reconfigurable systems that can realize rapid, well-controlled shape change. Herein we present a systematic, integrated experimental and computational study to control and tailor the multistable states of 3D structures and their reconfiguration paths. Our energy landscape analysis using a discrete shell model and minimum energy pathway methods leads to design maps for a controlled number of stable states by varying geometry and material parameters, and energy-efficient reconfiguration paths among the multistable states. Concurrently, our experiments show that 3D structures assembled from ferromagnetic composite thin films of diverse geometries can be rapidly reconfigured among their multistable states, with the number of stable states and reconfigurable paths in excellent agreement with computational predictions. In addition, we demonstrate a wide breadth of applications including reconfigurable 3D light emitting systems, remotely- controlled release of particles/drugs from a reconfigurable structure, and 3D structure arrays that can form desired patterns following the written path of a magnetic "pen". Our results represent a critical step towards the rational design and development of well-controlled, rapidly and remotely reconfigurable structures for many applications.
\end{abstract}




\section{Introduction}

Reconfigurable three-dimensional (3D) structures that can actively change their geometries and thereby their functionalities upon external stimuli (like mechanical forces, magnetic fields, hydration, and temperature $)^{1-4}$ are promising for a diverse range of applications including deployable solar panels $^{5}$, electromagnetic metamaterials ${ }^{6,7}$, phononics ${ }^{8-10}$, biomedical devices $^{11,12}$, soft robotics ${ }^{13-17}$, metasurfaces ${ }^{18-21}$, and many others. The design of reconfigurable structures usually relies on structural instabilities, stimuli-responsive constituent materials (like swellable gels and shape memory polymers $)^{22-24}$, or their heterogeneous combinations ${ }^{25}$. For example, origami and kirigami, ancient art of folding of 2D thin sheets along predefined creases to create $3 \mathrm{D}$ objects, have been widely used as platforms for deployable structures due to their multiple deployed stable states under mechanical deformation ${ }^{7,15,26-37}$. Structural instabilities have also been exploited in recently developed mechanics-guided assembly approaches to complex 3D functional architectures and electronics in a diversity of configurations and a broad range of material compositions ${ }^{38-42}$. Through elastically deforming the assembly platform in different time sequences (i.e., loading-path control), the 3D assembled architectures and electronics can morph into different shapes ${ }^{43-47}$. In addition to mechanical deformation and associated structural stability, reconfigurable structures can also be realized through active constituent materials including ferromagnetic composites ${ }^{3,48-50}$, hydrogels ${ }^{22}$, shape memory polymers (SMPs), liquid crystal elastomers (LCEs) ${ }^{51}$, and electrochemically active materials ${ }^{52}$, which can change their shape upon external stimuli.

Despite intensive studies, realizing robust and efficient architectural reconfiguration without the need for persistent external stimuli, especially for asymmetric and complicated modes, remains a challenge. Reconfigurability based on the multistability of structures can potentially realize shape changes without the requirement of persistent external stimuli ${ }^{53-56}$. However, there is still a lack of fundamental understanding of the principles that control the 
multistable states, where each stable state corresponds to a local minimum point in a highdimensional and complex energy landscape. One challenge is associated with the existence of a large number of local minimum configurations, which could affect the stability of the targeted stable state under perturbations (e.g., environmental noises) or trap the structure in an intermediate state during the process of reconfiguration. For example, it is recently recognized by researchers that hidden local minimum configurations can destroy the designed pathway of deploying origami structures ${ }^{28,30,57}$. Thus, it is crucial to be able to manipulate the energy well depth of targeted configurations and eliminate unfavorable local minima in the design of reconfigurable structures. Another challenge stems from the complicity of the transition paths from one local minimum state to another, especially when the two states are separated by other stable and transition states ${ }^{58-62}$. A recent work ${ }^{44}$ shows that the transition from a uniformly deformed cylindrical shell to a 9-dimple buckled pattern needs to pass 7 local minimum configurations, which cannot be achieved by directly applying local probes to the shell. Complicated transition paths among local minima are also found in mechanical metamaterials, which further provides new design parameters to program the deformed configurations of the structures $^{61}$. In addition, it is also very challenging to realize on-demand, locally controlled, and rapid reconfiguration of the deformed, highly nonlinear structures ${ }^{63,64}$. These challenges call for a new means of systematically surveying the energy landscapes of the multistable structures to probe and tailor the energy barrier height among different local minima.

In this work, we present a set of strategies and design concepts to address these challenges. 3D structures are assembled from ferromagnetic composite thin films using schemes that reply on strain release from biaxially prestrained elastomer platforms ${ }^{38,40}$. We show that the assembled 3D structures can be remotely and rapidly deformed into multiple distinct states under external magnetic forces and maintain the deformed shapes even after the magnetic field is removed. To identify the spectrum of existing multistable states, we conduct fundamental studies of the 
multistablity of the 3D structures through exploring their energy landscapes using a discrete shell mode ${ }^{65,66}$. We develop phase diagrams showing how the available stable states sensitively depend on the material and geometrical parameters of the elastic structures, which are validated by our experiments. Reconfiguration paths between the stable states are then identified using a combination of the string ${ }^{67}$ and binary image transition state search methods and realized by magnetically reconfiguring 3D structures assembled from ferromagnetic composite thin films. A wide range of geometries, including ribbons and structures that resemble tables, baskets, flowers, boxes, single and double beams, and their multistable states are demonstrated in both simulations and experiments. In addition, 3D reconfigurable light emitting systems illustrate the capacity to integrate other materials and functional components into these $3 \mathrm{D}$ reconfigurable structures. Magnetically actuated particle delivery in different modes of a table structure highlights applications in reconfigurable 3D systems of potential relevance in biomedical devices. Furthermore, a structure array of reconfigurable 3D ferromagnetic structures, which can display well-controlled patterns formed by reconfiguring the structures following the written path of a magnetic "pen", serves as an additional example in reconfigurable systems.

\section{Results and Discussions}

\section{Stability of multistable states of $3 D$ buckled structures}

Figure 1a schematically illustrates the assembly process of a 3D structure from ferromagnetic composite thin films via compressive buckling, followed by architectural reconfiguration via magnetic actuation. The ferromagnetic composite is prepared by homogeneously embedding hard $\mathrm{NdFeB}$ (neodymium-iron-boron) microparticles with an average diameter of $5 \mu \mathrm{m}$ into a soft elastomer, polydimethylsiloxane (PDMS) (Figure S1, Supporting Information). NdFeB is chosen for our study because its high residual magnetic flux density over a wide range of applied magnetic fields allows complex modes of reconfiguration ${ }^{68}$. The assembly of $3 \mathrm{D}$ ferromagnetic structure starts with patterning the ferromagnetic composite thin films (125 $\mu \mathrm{m}-150 \mu \mathrm{m}$ thick) into 2D layouts using a $\mathrm{CO}_{2}$ laser (VLS 3.50, University 
Laser System, Norman, OK), followed by their axial magnetization using impulse magnetic fields (about $2.7 \mathrm{~T}$ ) generated by an impulse magnetizer. The $2 \mathrm{D}$ patterns are then laminated onto a prestretched elastomer (Dragon skin, Smooth-on, Chicago, IL), with a very thin layer of superglue applied at selective locations (bonding sites) of the 2D patterns to generate strong bonding between the pattern and the elastomer substrate. The interfacial interactions at all other locations are dominated by comparatively weak van der Waals forces. Releasing the prestrain in the substrate leads to compressive forces on the $2 \mathrm{D}$ ferromagnetic pattern at the bonding sites and transforms the pattern into a 3D structure through controlled compressive buckling. Throughout the paper, we adopt a local coordinate system for the 3D structure such that the $2 \mathrm{D}$ layout is always on the $\mathrm{x}-\mathrm{y}$ plane with its normal vector as the z-direction. The assembled 3D structure can have multiple stable shapes, and the reconfiguration among them is achieved remotely and rapidly by leveraging external magnetic forces applied to the ferromagnetic films. Due to structural stability, the deformed shape can be well maintained after the magnetic force is removed.

Figure 1b presents experimental and corresponding computational results of a 3D ferromagnetic table structure (film thickness: $150 \mu \mathrm{m}$ ) and its multiple stable states. This table structure is oriented such that gravity is in the negative $z$-direction, perpendicular to the substrate, which is the case for most of the structures in this study unless specified otherwise. To efficiently explore the possible stable states existing in the table structure, we use the discrete shell model to locate the stable configurations, where the energy of the buckled structures is locally minimized. Three distinct states (state 1-3) with a total of nine configurations are discovered. By magnetically deforming the assembled table structure using a portable disk magnet (Neodymium magnet, K\&J Magnetics), in a way that one leg becomes flat and in contact with the substrate while the other three legs are in a buckled status, the table structure (state 1) can be reconfigured into distinct stable configurations with four different orientations (state 2: shape I-IV). Unlike previously reported reconfigurable 3D structures that rely on 
persistent external stimuli to maintain their deformed shape ${ }^{39,48,49,68-70}$, the deformed configurations of the table structure shown here can well maintain their shapes after the applied magnetic field is removed, due to structural stability (will be discussed in detail later). Magnetically deforming the structure with an additional leg of the table structure becoming flat and in contact with the substrate results in another stable state with four different orientations (state 3: shape I-IV). The discrete shell model (see description in the Methods section) captures all the stable states and their different orientations in experiments, with the color in the results denoting the displacement along the $\mathrm{z}$ direction in the structure.

Using the same experimental and computational strategy, we further study the effect of geometry and material properties on the entire spectrum of stable states, resulting in the phase diagram shown in Figure 1c. It illustrates the number of stable states according to two dimensionless parameters that correspond to the ratios between three relevant energy scales gravitational $\left(E_{G}\right)$, stretching $\left(E_{S}\right)$, and bending $\left(E_{B}\right)$. These energy scales can be expressed in terms of the material and geometric parameters of the 3D structure:

$$
\begin{aligned}
& E_{G}=\rho g L^{3} t, \\
& E_{S}=E L^{2} t, \\
& E_{B}=\frac{E t^{3}}{1-v^{2}},
\end{aligned}
$$

where $\rho$ is the material density, $E$ is the Young's modulus, $v$ is the Poisson ratio, $t$ is the film thickness, and $L$ represents the in-plane size of the structure (the width of legs is used for this study). The cubic term of $L$ in $E_{G}$ comes from the fact that the volume of the structure scales with $L^{2}$ and the displacement in the direction of gravity is proportional to $L$. Three regimes can be identified in Figure 1c: (i) 3 distinct stable states, (ii) 2 distinct stable states, and (iii) 1 distinct stable state, which are controlled by two dimensionless variables $E_{S} / E_{B}=$ $L^{2}\left(1-v^{2}\right) / t^{2}$ and $E_{G} / E_{B}=\rho g L^{3}\left(1-v^{2}\right) / E t^{2}$. The variable $E_{S} / E_{B}$ characterizes the bendability of the structure, with a larger value indicating that it is easier to generate bending 
deformation. The variable $E_{G} / E_{B}$ describes the competition between the gravitational and bending energies. When $E_{S} / E_{B}$ and $E_{G} / E_{B}$ are relatively large, three distinct stable states as shown in figure $1 \mathrm{~b}$ exist in regime $\mathrm{i}$, with states 2 and 3 having 4 different orientations. As the two dimensionless variables decrease, state 2 becomes unstable, resulting in two distinct stable states (states 1 and 3) for regime ii. When $E_{G} / E_{B}$ is further reduced to an even smaller value, only the buckled-up state (state 1) is stable in regime iii. To validate the phase diagram experimentally, we fabricate 3D ferromagnetic structures of the same film thickness $(t=125$ $\mu \mathrm{m})$ and material properties $(\rho, E$, and $v)$ but of different sizes, and magnetically reconfigured them into their multistable states. Figure 1d (i-iii) demonstrate the number and configurations of the stable states for structures with $L=1.04 \mathrm{~mm}, 0.8 \mathrm{~mm}$, and $0.5 \mathrm{~mm}$, respectively, which agree well with the predicted design phase diagram. Please note that the overall in-plane dimensions of the structures are scaled proportionally with $L$ as it varies. The design diagram provides very important guidelines for achieving a controlled number of stable states or for removing undesired stable states by tuning the geometry and material properties of the structure for various applications. For instance, state 2 may be undesirable for specific applications, in which case the structure should be designed such that it falls within region ii, where only states 1 and 3 are stable.

\section{Transition paths among distinct stable states of 3D buckled structures}

Identifying the optimal transition pathways between stable states of a $3 \mathrm{D}$ structure is critical for well-controlled, energy-efficient reconfiguration. We further examine the energy landscape of the table structure to identify the so-called minimum energy pathways, corresponding to steepest-descent pathways between metastable states in which the maximum energy along the path occurs at a saddle point (the transition state). We find the observed variations in the transition pathways to progress from state 3 to state 1 of the table structures can be broadly grouped into three regions shown in Figure 2a, as we vary the in-plane size of 
the structures, $L$, but keep their material properties $(\rho, E$, and $v)$ and film thickness $(t=125 \mu \mathrm{m})$ constant. The marked red and blue points correspond to those in the 3-state and 2-state regimes identified in the design diagram in Figure 1c, respectively.

For $L>0.89 \mathrm{~mm}$, two independent minimum energy pathways are found and illustrated in Figure $\mathbf{2 b}$ for a representative table structure with $L=1.04 \mathrm{~mm}$ : a direct pathway, with energy barrier $\Delta E_{1}$ (path 1), and a path that passes through state 2 as an intermediary, with energy barriers $\Delta E_{2}$ between states 3 and 2 and $\Delta E_{3}$ between states 2 and 1 (path 2). Snapshots of the configurations from simulations and magnetic reconfiguration experiments along the pathways and the complete structural transformation process of the table structure are shown in Figure 2c and Supplementary Movies 1 and 2, respectively. The energy barriers of the pathways demonstrate both the ease of transitioning between the states and their stability. For example, the energy barrier to progress from state 3 to state 1 is much smaller than that from the inverse direction, so state 1 is more stable. Comparing paths 1 and 2 also suggests that a smaller input energy is needed when reconfiguring the table structure along path 2 (passing two saddle points) than that along path 1 . However, the finite energy barrier $\Delta E_{3}$ also indicates that the transition can be potentially trapped in state 2 if insufficient external energy is provided for reconfiguration. In addition, reconfiguration along path 2 requires more variance in the direction and the strength of external magnetic forces, compared to the direct pathway following path 1 . These complexities clearly show the importance of harnessing the energy landscape analysis for guiding the choice of transition paths based on specific applications and the nature of available external magnetic fields (or other external stimuli). In addition, it is also worth noting that the same pathways are observed in experiments when an external magnetic field is introduced to reconfigure the structure from state 3 to state 1, which indicates the high fidelity and reliability of the approach used in this study. Moreover, the reconfigurations in our experiment can be completed within a few seconds (Supplementary Movies 1 and 2). Such 
fast and remotely controlled transitions are desired for numerous applications, such as in soft robotics $^{13-15,71}$ and multifunctional metasurfaces ${ }^{72,73}$.

As we decrease $L$, all the energy barriers become smaller monotonically, as shown in Figure 2a. At $L=0.89 \mathrm{~mm}$, we cross the boundary between the 3-state and the 2-state regimes in the phase diagram, and we find that $\Delta E_{3}$ reduces to zero, a signature that state 2 is no longer stable. For $0.78 \mathrm{~mm}<L<0.89 \mathrm{~mm}$, there remains two distinct minimum energy pathways to transition from state 3 to state 1 , even in the absence of a stable state 2 . The typical energy profiles for this range of $L$ values are illustrated in Figure $\mathbf{2 b}$ for $L=0.80 \mathrm{~mm}$. Snapshots of the configurations from simulations and experiments for $L=0.80 \mathrm{~mm}$ are further shown in Figure 2d, with the full pathways provided in Supplementary Movies 3 and 4. The shape evolutions along the pathways actually remain qualitatively similar to those shown in Figure 2c. However, we use the superscript * in path $2 *$ in Figure $2 \mathbf{d}$ to reflect that state 2 is no longer stable, unlike for path 2 in Figure 2c. Interestingly, we also find that there is a crossover in the preferred pathways at $L=0.84 \mathrm{~mm}$, where $\Delta E_{1}=\Delta E_{2}$ in Figure 2a. For a larger $L(0.84 \mathrm{~mm}$ $<L<0.89 \mathrm{~mm}$ ), path $2 *$ is favorable because the corresponding energy barrier is smaller, while for smaller $L(0.78 \mathrm{~mm}<L<0.84 \mathrm{~mm})$, path 1 has a lower energy barrier.

The preference for path 1 at smaller $L$ values is further accentuated by the loss of path $2^{*}$ as a minimum energy pathway for $0.56 \mathrm{~mm}<L<0.78 \mathrm{~mm}$. For this range of $L$ values, we are unable to identify path $2 *$ in simulations, as indicated by the lack of data points in Figure 2a. The absence of path $2 *$ is further demonstrated by our failure to experimentally reconfigure the table structure along this pathway using the portable disk magnet (see Supplementary Movie 6), unlike the case for larger $L$. Here, only path 1 remains. The typical energy profile for the transition along path 1 in this range of $L$ values is shown in Figure $\mathbf{2 b}$ for $L=0.72 \mathrm{~mm}$, with the corresponding snapshots of the reconfiguration and the full pathway given in Figure 2e and Supplementary Movie 5, respectively. 
State 3 itself becomes unstable when $L$ is further reduced below $0.56 \mathrm{~mm}$, where we enter the 1-state regime. At this point, we cross the phase boundary between the 2-state and 1state regimes, and the barrier $\Delta E_{1}$ becomes zero in Figure 2a. Taken together, these highly nonlinear behaviors observed for the transition pathways make the energy landscape analysis a necessary tool for an efficient and robust design of the multistable configurations and the transition paths among them.

\section{$3 D$ multistable structures of diverse configurations}

The assembly strategy and the energy landscape analysis for multistability described above are versatile and can be extended to other geometries and types of structures, as illustrated in Figure 3 and Figure S2. Figure 3a shows an assembled ferromagnetic basket structure (shape I; film thickness: $125 \mu \mathrm{m}$ ) and its multiple stable states (shapes II-IV) that can be achieved via magnetic control. In addition, by introducing creases to selective locations of a ribbon structure (crease thickness: $70 \mu \mathrm{m}$; non-crease thickness: $125 \mu \mathrm{m}$ ), we can create an origami structure, which can be further tuned to display multiple stable configurations by using magnetic forces.

The elastomer substrate used for 3D assembly in Figure 1, Figure 2 and Figure 3a provides essential support for the assembled 3D structures, but it also poses some limitations for the reconfiguration in the out of plane direction. To allow more freedom for spatial reconfiguration, the substrate underneath the $3 \mathrm{D}$ table structure is removed, while the substrate adjacent to the bonding sites is maintained to support the 3D structure (see Figure S3). Such 3D structure with hollow substrates allows bending up/down deformations across the plane of the substrate. Figure 3b and Figure $\mathbf{S 2}$ demonstrate a rich library of reconfigurable 3D structures on hollow substrates, including basket, single- and double-beam structures, as well as their distinct, multi-stable shapes. 
It is very interesting to note that the multi-stable configurations on hollow substates are sensitive to the orientation of the structures. Take the table structure for example, it is shown that the structure only displays two stable states, buckled up and down states, when placed horizontally (i.e., the direction of gravity is perpendicular to the in-plane direction of the substrate, Figure S2d). However, when the table structure is placed vertically with the direction of gravity in the $x$-direction (Figure 3b), in addition to the buckled up (Shape I) and down (Shape IV) states, it can deform into an asymmetric, twisted shape (Shape II), similar to state 3 of the table structure with an intact substrate. This deformed shape can be further magnetically deformed into shape III, with the four bonding sites attached to the substrate and the rest of the structure self-supported in the air. We also carry out energy landscape analysis for this structure, the details of which are presented in Figure S4. Similar to the case with an intact substrate, we can distinguish several regimes with different numbers of stable states, depending on the three relevant energy scales: gravitational, stretching, and bending energies. Furthermore, the dimension of the structure on intact and hollow substrates can be further reduced using $\mathrm{CO}_{2}$ laser (Figure S5), and the structures are shown to maintain their shapes after the applied magnetic field is removed. Structures at an even smaller scale can be achieved using more advanced microfabrication techniques. However, miniaturization of these structures is not the focus of this study and will be pursued elsewhere.

In addition to tuning the strength and the direction of external magnetic forces for reconfiguration, an alternative and potentially more versatile means to realize magnetically controlled reconfiguration is through tuning the distribution of magnetically active materials within hybrid 3D structures. Figure 3c shows reconfigurable hybrid 3D table and flower structures by locally integrating ferromagnetic composite film patterns (125 $\mu \mathrm{m}$ thick) onto a PDMS layer (180 $\mu \mathrm{m}$ thick) (Method I, Figure S6). Both hybrid 3D structures are magnetically tuned to display five stable configurations using a portable magnet and maintain them after the magnetic force is removed, the capabilities of which are similar to those made of pure 
ferromagnetic materials. Hybrid 3D structures at a smaller scale can be achieved by directly patterning a bilayer or multilayer 2D structure using the raster mode of a laser or reactive ion etching (Method II in Figure S6) to remove ferromagnetic films at undesired regions. In addition, hybrid 3D structures can potentially allow more local magnetic control by varying the direction and the strength of the residual magnetic field in each ferromagnetic pattern through magnetization in different angles and mixing different concentrations of magnetic particles in the composite film, respectively.

\section{Applications of $3 D$ multistable structures}

Integrating functional materials and components onto 2D ferromagnetic composite patterns provides access to 3D tunable, functional devices. As an example, we demonstrate reconfigurable light emitting systems in Figure 4a. The fabrication begins with the integration of two light emitting diodes (LEDs) with a dimension of $1 \mathrm{~mm} \times 0.4 \mathrm{~mm} \times 0.2 \mathrm{~mm}$ onto designated locations of a double-beam structure. Copper thin films (thickness: $\sim 60 \mu \mathrm{m}$ ) connected to the two poles of the LED serve as interconnects for subsequent LED activation. Formation of 3D architectures from these 2D functional precursors follows the 3D buckling schemes introduced earlier. Magnetically tuning the assembled 3D structure to deform from shape I to II (or III) enables a firm contact between the two poles of the LED (located at one beam) with two corresponding copper films (on the substrate) that are connected to an external power supply for power input, and therefore activates the LED. Furthermore, the LED can maintain its "on" status after the magnetic force is removed due to the stability of the deformed configurations (shapes II and III). When the two beams are simultaneously deformed to form stable configuration IV using a magnet, both LEDs are turned on. This simple example suggests a broad range of possibilities in other types of electronic and optoelectronic devices.

The reconfigurable structures presented in this work also have a lot of potential applications in soft robotics, metasurfaces, and biomedical devices. Based on the magnetically 
actuated 3D reconfiguration strategy, we also assemble a functional structure in a table shape for the delivery of particles/drugs in a well-controlled manner. As shown in Figure $\mathbf{4 b}$ and Figures S7, the device is made from a table structure in ferromagnetic composite films (thickness: $180 \mu \mathrm{m}$ ) and a thin layer of pure PDMS film (thickness: $60 \mu \mathrm{m}$ ) in a concave shape as the table face, where a silica particle is placed for the demonstration. To trigger the delivery of the particle, we used a portable magnet to enable the deformation of the table structure to release the particle. In addition, the magnet is manipulated to enable the deformation of the structure into multi-stable configurations with 4 different orientations (modes I-IV), leading to particle delivery in 4 controlled directions. The layout of the hybrid ferromagnetic table allows the table face that supports the particle to stay insensitive to the applied magnetic field when the table structure is magnetically actuated. In addition, the soft, thin PDMS layer does not influence the reconfiguration of the whole table structure. Such flexible, remotely reconfigurable structure has a lot of potential applications in biomedical devices.

To demonstrate the scalability of the fabrication process as well as more capabilities of reconfigurable structures, we assembled a $4 \times 4$ structure array of reconfigurable table structures with a unit cell size of $3 \times 3 \mathrm{~mm}^{2}$. Each structure unit has the identical original shape, as shown in the magnified image (image A) in Figure 5. Using a portable magnet to tune the 3D structure array following a defined path will consecutively and rapidly reconfigure the 3D structure along the path into a deformed shape (image B) that can be maintained after the magnet passes. Following different paths leads to varied magnetically drawn patterns, like the "V" and line patterns shown in Figure 5a and Figure S8, respectively. Also, each structure unit is allocated sufficiently far to avoid interference during magnetic control, i.e., the structure unit is actuated consecutively along the path. Figure $5 \mathrm{~b}$ shows a $4 \times 5$ structure array of table structures on hollow substrates. The structure can be reconfigured from its "popped down" (image C) configuration to its "popped up" (image D) one following the path of the magnet to display desired patterns like letter "M" (Supplementary Movie 7). 


\section{Discussion}

To conclude, we have tightly integrated experiments and modelling to control and tailor multi-stable states existing in highly nonlinear 3D ferromagnetic structures as well as energyefficient reconfiguration paths among them for the development of reconfigurable systems. Our systematic computational energy landscape analysis predicts a design map for a targeted number of multi-stable configurations by varying material properties and geometries, as well as efficient transition paths among the distinct stable states. These predictions are thoroughly validated by our experiments of fabricating multistable 3D ferromagnetic structures and magnetically reconfiguring the structures among their multistable states following different pathways. Using this strategy, a plethora of symmetric and asymmetric 3D structures of diverse geometries are fabricated and rapidly reconfigured among their multistable states, including those that resemble ribbons, tables, baskets, flowers, boxes, single and double beams. Furthermore, the integration of electronic components onto ferromagnetic composites provides immediate access to reconfigurable 3D functional devices, which is demonstrated in reconfigurable light emitting systems. The development of the magnetically actuated table structure for particle/drug delivery highlights capabilities of potential relevance to smart, remotely controlled biomedical devices. Arrays of 3D structures that are deformed consecutively to display desired patterns suggest opportunities in reconfigurable metasurfaces. We believe our fundamental studies of the multistability of 3D structures establish the foundations for robust and reproducible architectural reconfiguration behaviors. More broadly, our integrated experimental and modelling approach can be applied to the design and development of diverse types of reconfigurable structures and systems, including origami, kirigami and stimuli-responsive structures, where the exploration of energy landscapes and the tunability of multistable states are needed but not systematically analyzed. The enabled reconfigurable structures and functional systems are applicable for wide-ranging applications, such as in deployable solar panels, photonics, morphable architected materials, and soft robotics. 


\section{Methods}

Fabrication of 3D ferromagnetic composite structures. Ferromagnetic composite was prepared by homogeneously mixing $\mathrm{NdFeB}$ (neodymium iron boron) microparticles with an average diameter of $5 \mu \mathrm{m}$ (MQFP-B-2007609-089, Neo Magneuench) into uncured Polydimethylsiloxane (PDMS) resin at a volume ratio of 1:5 using a planetary mixer (AR-100, Thinky) at $2000 \mathrm{rpm}$ for $2 \mathrm{~min}$, followed by defoaming at $2000 \mathrm{rpm}$ for $1 \mathrm{~min}$. The evenly mixed ferromagnetic composite was spin coated at $500 \mathrm{rpm}$ for $15 \mathrm{~s}$ and completely cured at room temperature. The obtained ferromagnetic composite film was then patterned into desired geometries using a $\mathrm{CO}_{2}$ laser and laminated onto a prestreched soft silicone elastomer substrate (Dragon Skin; Smooth-On, Easton, PA) as an assembly platform. A thin layer of superglue was applied onto selective sites of the back surface of the pattern to form strong adhesion only at the bonding sites. Releasing prestrain in the elastomer substrate led to geometry transformation of the $2 \mathrm{D}$ pattern into a $3 \mathrm{D}$ structure.

Actuation of 3D structures. An axially magnetized cylinder neodymium magnet with a diameter of $1.27 \mathrm{~cm}$ (D8Y0, K\&J magnetics) was used to reconfigure 3D structures among their distinct multistable states. The strength and the direction of the applied magnetic field were adjusted/controlled via changing the distance and the angle between the circular magnet surface and 3D ferromagnetic structures.

Fabrication of 3D light emitting devices. The fabrication of light emitting devices began with integrating two rectangular $\mathrm{Cu}$ films, separated with a distance of $6 \mathrm{~mm}$ as conductive traces, at selective locations of the $2 \mathrm{D}$ ferromagnetic composite precursor using a thin layer of super glue. This is followed by adding a LED (dimension: $1 \mathrm{~mm} \times 0.4 \mathrm{~mm} \times 0.2 \mathrm{~mm}$ ) on top of the $\mathrm{Cu}$ films with conductive epoxy (Allied Electronics Corp., Fort Worth, TX), with the two poles of the LED aligned with the corresponding copper films. Covering the contact area between the 
LED and the $\mathrm{Cu}$ films with transparent, nonconductive epoxy completed the integration. The functional 2D precursor was then assembled into a 3D architecture following the previously introduced compressive buckling technique. The 3D architecture was then magnetically deformed into a stable state that enables the contact of the $\mathrm{Cu}$ traces connected to the LED with two conductive $\mathrm{Cu}$ films (on the substrate; connected to a power supply), leading to the activation of the LED. In addition, the location and the number of LEDs that are actuated were well controlled with different stable states of the 3D structure. More importantly, the LEDs can maintain their "on" status after the magnetic field is removed, due to the mechanical stability of the structure.

Fabrication of a robot for particle release. A thin layer of a pure PDMS film (thickness: 60 $\mu \mathrm{m})$ was laser cut and attached to the top of the table structure to form a semi-spherical shape for holding particles, as can be seen in Figure S7.

Fabrication of reconfigurable structure array. 3D table structures were assembled into an array with a distance of $2 \mathrm{~cm}$ between two table structures to ensure that magnetic manipulation of one structure unit does not affect its adjacent structures. The magnet was manually moved from one targeted structure to another following the specified trajectory including a line and an alphabet pattern to form the desired patterns.

\section{Modeling:}

Discrete shell model ${ }^{65,66}$. The structures are modelled as a thin elastic sheet using a 2D Delaunay triangulation. The structure energy has four contributions, corresponding to stretching, bending, gravity, and a repulsive substrate interaction. The stretching energy is given by the summation of the stretching energies of the individual bonds in the triangulation, each with a stiffness related to the Young's modulus, $E$, and material thickness, $t$, 


$$
E_{\text {stretch }}=\sum_{b} \frac{\sqrt{3}}{4} E t\left(r_{b}-r_{b}^{0}\right)^{2}
$$

where $r_{b}$ and $r_{b}^{0}$ are the length and relaxed length for bond $b$. The bending energy is obtained by considering pairs of adjacent faces to be connected by elastic hinges, where the optimal dihedral angle is $\pi$. The stiffness of the hinge is again related to the material parameters, but also upon the size and shape of the two faces so that it accurately approximates the flexural rigidity of continuum elastic models. The result is

$$
E_{\text {bend }}=\frac{E t^{3}}{12\left(1-v^{2}\right)} \sum_{h} \frac{r_{h}}{A_{h, 1}+A_{h, 2}}\left(1+\cos \theta_{h}\right)
$$

where $v$ is the Poisson ratio, $r_{h}$ is the length of hinge $h, A_{h, i}$ is the area of each triangular face, and $\theta_{h}$ is the dihedral angle of the hinge. The gravitational energy is simply obtained by a summation of the gravitational energies of each of the triangular segments. The heights of which are measured relative to the center of the flat substrate in a direction dependent upon the orientation of the structure. Finally, in the cases with a substrate the repulsive interaction is modelled using a Lennard-Jones 9-3 potential that is cutoff and shifted to remove the attractive region. Consequently, the model does not take into consideration the effect of adhesion which may become significant at small scales.

In our simulations, we set the Young's modulus of the ferromagnetic film to be $E=$ 1.4 $\mathrm{MPa}^{48}$ and the film density to its measured experimental value of $\rho=2.59 \mathrm{~g} / \mathrm{cm}^{3}$. It is worth noting that the current discrete shell leads to a fixed Poisson ratio for the in-plane deformation of $v=1 / 3^{74}$. However, since the deformation is mainly determined by the bending and stretching stiffness, the smaller Poisson ratio compared to most polymeric materials $(\sim 0.5)$ will not significantly influence the simulations. This can be validated through the excellent agreement between the simulations and experiments. For the simulated structures, we first generate triangular mesh, with an average mesh size of $0.1 \mathrm{~mm}$, based on the CAD files used for manufacturing the experimental samples. 
Finding the stable states. Each structure is initialized by steadily moving the binding sites from their positions in the flat precursor to their final positions. Random forces are applied to different regions of the structure so that it will buckle in different directions, and the structure is relaxed until the energy is minimized. This process is repeated to identify all the different local minima in the energy landscape.

Finding the reconfiguration pathways. These are computed by the minimum energy paths (MEP). Between any two states in the energy landscape, the MEP is defined as a path in which the gradient of the energy is parallel to the path tangent vector. The MEP will also pass through at least one saddle point in the landscape, a local energy maximum along the pathway. To locate the MEPs efficiently, a combination of two methods are utilized. First, the transition state is found using the binary image transition state search (BITSS). The BITSS method involves two states, $\boldsymbol{x}_{1}, \boldsymbol{x}_{2}$, that begin at the two local minima and are minimized according to the augmented potential,

$$
E_{T O T}\left(\boldsymbol{x}_{1}, \boldsymbol{x}_{2}\right)=E\left(\boldsymbol{x}_{1}\right)+E\left(\boldsymbol{x}_{2}\right)+k_{e}\left(E\left(\boldsymbol{x}_{1}\right)-E\left(\boldsymbol{x}_{2}\right)\right)^{2}+k_{d}\left(\left\|\boldsymbol{x}_{1}-\boldsymbol{x}_{2}\right\|-d_{0}\right)^{2},
$$

where the first two terms are the energies of the two states, and the third and fourth are constraints that are parametrized by $k_{e}$ and $k_{d}$. The energy constraint penalizes any difference between the two states to ensure that neither state passes over the division between the basins of attraction for the two minima. The second constraint prescribes the distance between the states to be $d_{0}$, which is steadily decreased to zero so that the two states converge towards the transition state. Once a transition state for a given structure is found, the method of continuation can be exploited to locate the corresponding transition state for structures with different material properties. The pathways themselves are then found using the string method, initialized using 
the known minima and transition states. While the string method itself can in principle be harnessed to find the transition states, we find the BITSS method is faster and more accurate.

\section{Acknowledgements}

We acknowledge the support of the following grants: NSF-CMMI-2020676 (X.W.), NSFCMMI-2020476 (T. Z.). S.J.A. is supported by a studentship from the Engineering and Physical Sciences Research Council (EPSRC). Simulations were performed at the facilities of the Hamilton HPC Service of Durham University and the Comet cluster (Award TG-MSS170004 to T.Z.) in The Extreme Science and Engineering Discovery Environment.

\section{Competing interests}

The authors declare no competing financial interests.

\section{References}

1 Overvelde, J. T. B., Weaver, J. C., Hoberman, C. \& Bertoldi, K. Rational design of reconfigurable prismatic architected materials. Nature 541, 347-352, doi:10.1038/nature20824 (2017).

2 Filipov, E. T., Tachi, T. \& Paulino, G. H. Origami tubes assembled into stiff, yet reconfigurable structures and metamaterials. Proceedings of the National Academy of Sciences 112, 12321-12326, doi:10.1073/pnas.1509465112 (2015).

3 Kim, Y., Yuk, H., Zhao, R. K., Chester, S. A. \& Zhao, X. H. Printing ferromagnetic domains for untethered fast-transforming soft materials. Nature 558, 274-279, doi:10.1038/s41586-018-0185-0 (2018).

4 Wehner, M. et al. An integrated design and fabrication strategy for entirely soft, autonomous robots. Nature 536, 451-455, doi:10.1038/nature19100 (2016).

5 Thesiya, D., Srinivas, A. \& Shukla, P. A novel lateral deployment mechanism for segmented mirror/solar panel of space telescope. Journal of Astronomical Instrumentation 4, 1550006 (2015).

6 Fu, Y. H. et al. A Micromachined Reconfigurable Metamaterial via Reconfiguration of Asymmetric Split - Ring Resonators. Advanced Functional Materials 21, 3589-3594 (2011).

7 Wang, Z. et al. Origami - based reconfigurable metamaterials for tunable chirality. Advanced Materials 29, 1700412 (2017).

8 Babaee, S., Overvelde, J. T. B., Chen, E. R., Tournat, V. \& Bertoldi, K. Reconfigurable origami-inspired acoustic waveguides. Sci Adv 2, doi:ARTN e160101910.1126/sciadv.1601019 (2016).

9 Babaee, S., Viard, N., Wang, P., Fang, N. X. \& Bertoldi, K. Harnessing deformation to switch on and off the propagation of sound. Advanced Materials 28, 1631-1635 (2016). 
10 Deng, B., Wang, P., Tournat, V. \& Bertoldi, K. Nonlinear transition waves in freestanding bistable chains. Journal of the Mechanics and Physics of Solids, 103661 (2019).

11 Damdam, A., Qaisar, N. \& Hussain, M. M. Honeycomb-serpentine silicon platform for reconfigurable electronics. Applied Physics Letters 115, 112105 (2019).

12 Anderson, P. L., Mahoney, A. W. \& Webster, R. J. Continuum Reconfigurable Parallel Robots for Surgery: Shape Sensing and State Estimation With Uncertainty. Ieee Robot Autom Let 2, 1617-1624, doi:10.1109/Lra.2017.2678606 (2017).

13 Shepherd, R. F. et al. Multigait soft robot. P Natl Acad Sci USA 108, 20400-20403, doi:10.1073/pnas.1116564108 (2011).

14 Plitea, N., Lese, D., Pisla, D. \& Vaida, C. Structural design and kinematics of a new parallel reconfigurable robot. Robot Cim-Int Manuf 29, 219-235, doi:10.1016/j.rcim.2012.06.001 (2013).

15 Tang, Y., Li, Y., Hong, Y., Yang, S. \& Yin, J. Programmable active kirigami metasheets with more freedom of actuation. Proceedings of the National Academy of Sciences 116, 26407-26413 (2019).

16 Pandey, A., Moulton, D. E., Vella, D. \& Holmes, D. P. Dynamics of snapping beams and jumping poppers. Epl-Europhys Lett 105, doi:Artn 2400110.1209/02955075/105/24001 (2014).

17 Gorissen, B., Melancon, D., Vasios, N., Torbati, M. \& Bertoldi, K. Inflatable soft jumper inspired by shell snapping. Sci Robot 5 (2020).

18 Liu, K., Tachi, T. \& Paulino, G. H. Invariant and smooth limit of discrete geometry folded from bistable origami leading to multistable metasurfaces. Nat Commun 10, doi:ARTN 423810.1038/s41467-019-11935-x (2019).

19 Wang, Q. et al. Optically reconfigurable metasurfaces and photonic devices based on phase change materials. Nature Photonics 10, 60 (2016).

20 Huang, C. et al. Reconfigurable metasurface for multifunctional control of electromagnetic waves. Advanced Optical Materials 5, 1700485 (2017).

21 Nemati, A., Wang, Q., Hong, M. \& Teng, J. Tunable and reconfigurable metasurfaces and metadevices. Opto-Electronic Advances 1, 180009 (2018).

22 Gladman, A. S., Matsumoto, E. A., Nuzzo, R. G., Mahadevan, L. \& Lewis, J. A. Biomimetic 4D printing. Nat Mater 15, 413-418 doi:10.1038/Nmat4544 (2016).

23 Lendlein, A., Jiang, H. Y., Junger, O. \& Langer, R. Light-induced shape-memory polymers. Nature 434, 879-882, doi:10.1038/nature03496 (2005).

24 Kim, J., Hanna, J. A., Byun, M., Santangelo, C. D. \& Hayward, R. C. Designing responsive buckled surfaces by halftone gel lithography. Science 335, 1201-1205 (2012).

25 Ding, Z. et al. Direct 4D printing via active composite materials. Sci Adv 3, doi:ARTN e160289010.1126/sciadv.1602890 (2017).

26 Peraza-Hernandez, E. A., Hartl, D. J., Malak, R. J. \& Lagoudas, D. C. Origami-inspired active structures: a synthesis and review. Smart Mater Struct 23, doi:Artn 094001 10.1088/0964-1726/23/9/094001 (2014).

27 Silverberg, J. L. et al. Using origami design principles to fold reprogrammable mechanical metamaterials. Science 345, 647-650, doi:10.1126/science.1252876 (2014).

$28 \mathrm{Na}$, J. H. et al. Programming reversibly self - folding origami with micropatterned photo - crosslinkable polymer trilayers. Advanced Materials 27, 79-85 (2015).

29 Filipov, E. T., Tachi, T. \& Paulino, G. H. Origami tubes assembled into stiff, yet reconfigurable structures and metamaterials. P Natl Acad Sci USA 112, 12321-12326, doi:10.1073/pnas.1509465112 (2015).

30 Silverberg, J. L. et al. Origami structures with a critical transition to bistability arising from hidden degrees of freedom. Nat Mater 14, 389 (2015). 
31 Tang, Y. C. et al. Design of Hierarchically Cut Hinges for Highly Stretchable and Reconfigurable Metamaterials with Enhanced Strength. Advanced Materials 27, 71817190, doi:10.1002/adma.201502559 (2015).

32 Li, S., Fang, H. \& Wang, K. W. Recoverable and Programmable Collapse from Folding Pressurized Origami Cellular Solids. Phys Rev Lett 117, doi:ARTN 11430110.1103/PhysRevLett.117.114301 (2016).

33 Dias, M. A. et al. Kirigami actuators. Soft Matter 13, 9087-9092, doi:10.1039/c7sm01693j (2017).

34 Fang, H. B., Li, S. Y., Ji, H. M. \& Wang, K. W. Dynamics of a bistable Miura-origami structure. Phys Rev E 95, doi:ARTN 05221110.1103/PhysRevE.95.052211 (2017).

35 Fang, H. B., Wang, K. W. \& Li, S. Y. Asymmetric energy barrier and mechanical diode effect from folding multi-stable stacked-origami. Extreme Mech Lett 17, 7-15, doi:10.1016/j.eml.2017.09.008 (2017).

36 Yang, Y., Dias, M. A. \& Holmes, D. P. Multistable kirigami for tunable architected materials. Phys Rev Mater 2, doi:ARTN 11060110.1103/PhysRevMaterials.2.110601 (2018).

37 Lele, A., Deshpande, V., Myers, O. \& Li, S. Snap-through and stiffness adaptation of a multi-stable Kirigami composite module. Composites Science and Technology 182, 107750 (2019).

$38 \mathrm{Xu}, \mathrm{S}$. et al. Assembly of micro/nanomaterials into complex, three-dimensional architectures by compressive buckling. 347, 154-159 (2015).

39 Zhang, Y. H. et al. A mechanically driven form of Kirigami as a route to 3D mesostructures in micro/nanomembranes. P Natl Acad Sci USA 112, 11757-11764, doi:10.1073/pnas.1515602112 (2015).

40 Wang, X. J. et al. Freestanding 3D Mesostructures, Functional Devices, and ShapeProgrammable Systems Based on Mechanically Induced Assembly with Shape Memory Polymers. Advanced Materials 31, doi:ARTN 180561510.1002/adma.201805615 (2019).

41 Jang, K. I. et al. Self-assembled three dimensional network designs for soft electronics. Nat Commun 8, doi:ARTN 1589410.1038/ncomms15894 (2017).

42 Lee, W. et al. Two-dimensional materials in functional three-dimensional architectures with applications in photodetection and imaging. Nat Commun 9, doi:ARTN 1417 10.1038/s41467-018-03870-0 (2018).

$43 \mathrm{Fu}, \mathrm{H}$. R. et al. Morphable 3D mesostructures and microelectronic devices by multistable buckling mechanics. Nat Mater 17, 268-276, doi:10.1038/s41563-0170011-3 (2018).

44 Panter, J., Chen, J., Zhang, T. \& Kusumaatmaja, H. Harnessing energy landscape exploration to control the buckling of cylindrical shells. Communications Physics 2, 19 (2019).

45 Zhao, H. B. et al. Buckling and twisting of advanced materials into morphable 3D mesostructures. P Natl Acad Sci USA 116, 13239-13248, doi:10.1073/pnas.1901193116 (2019).

46 Bai, K. et al. Geometrically reconfigurable 3D mesostructures and electromagnetic devices through a rational bottom-up design strategy. Sci Adv 6, eabb7417 (2020).

47 Pang, W. B. et al. Electro-mechanically controlled assembly of reconfigurable 3D mesostructures and electronic devices based on dielectric elastomer platforms. Natl Sci Rev 7, 342-354, doi:10.1093/nsr/nwz164 (2020).

48 Kim, Y., Parada, G. A., Liu, S. D. \& Zhao, X. H. Ferromagnetic soft continuum robots. Sci Robot 4, doi:ARTN eaax732910.1126/scirobotics.aax7329 (2019).

49 Hu, W. Q., Lum, G. Z., Mastrangeli, M. \& Sitti, M. Small-scale soft-bodied robot with multimodal locomotion. Nature 554, 81-85, doi:10.1038/nature25443 (2018). 
50 Boncheva, M. et al. Magnetic self-assembly of three-dimensional surfaces from planar sheets. P Natl Acad Sci USA 102, 3924-3929, doi:10.1073/pnas.0500807102 (2005).

51 Jampani, V. S. R. et al. Liquid crystal elastomer shell actuators with negative order parameter. Sci $A d v$ 5, doi:ARTN eaaw247610.1126/sciadv.aaw2476 (2019).

52 Xia, X. X. et al. Electrochemically reconfigurable architected materials. Nature 573, 205-213, doi:10.1038/s41586-019-1538-z (2019).

53 Song, Y. P. et al. Additively manufacturable micro-mechanical logic gates. Nat Commun 10, doi:ARTN 88210.1038/s41467-019-08678-0 (2019).

54 Yang, H. \& Ma, L. Multi-stable mechanical metamaterials by elastic buckling instability. J Mater Sci 54, 3509-3526, doi:10.1007/s10853-018-3065-y (2019).

55 O'Donnell, M. P., Towes, M., Groh, R. M. J. \& Chenchiah, I. V. Exploring Adaptive Behavior of Non-linear Hexagonal Frameworks. Front Mater 7, doi:ARTN 64 10.3389/fmats.2020.00064 (2020).

56 Li, Y. \& Pellegrino, S. A Theory for the Design of Multi-Stable Morphing Structures. Journal of the Mechanics and Physics of Solids 136, doi:ARTN 103772 10.1016/j.jmps.2019.103772 (2020).

57 Zhai, Z. R., Wang, Y. \& Jiang, H. Q. Origami-inspired, on-demand deployable and collapsible mechanical metamaterials with tunable stiffness. P Natl Acad Sci USA 115, 2032-2037, doi:10.1073/pnas.1720171115 (2018).

58 Groh, R. M. J. \& Pirrera, A. On the role of localizations in buckling of axially compressed cylinders. $P$ Roy Soc a-Math Phy 475, di:ARTN 2019000610.1098/rspa.2019.0006 (2019).

59 Virot, E., Kreilos, T., Schneider, T. M. \& Rubinstein, S. M. Stability Landscape of Shell Buckling. Phys Rev Lett 119, doi:ARTN 22410110.1103/PhysRevLett.119.224101 (2017).

60 Marthelot, J., Jimenez, F. L., Lee, A., Hutchinson, J. W. \& Reis, P. M. Buckling of a Pressurized Hemispherical Shell Subjected to a Probing Force. J Appl Mech-T Asme 84, doi:Artn 12100510.1115/1.4038063 (2017).

61 Medina, E., Farrell, P. E., Bertoldi, K. \& Rycroft, C. H. Navigating the landscape of nonlinear mechanical metamaterials for advanced programmability. Phys Rev B 101, doi:ARTN 06410110.1103/PhysRevB.101.064101 (2020).

62 Panter, J. R., Chen, J., Zhang, T. \& Kusumaatmaja, H. Harnessing energy landscape exploration to control the buckling of cylindrical shells. Communications Physics 2 , doi:ARTN 15110.1038/s42005-019-0251-4 (2019).

$63 \mathrm{Fu}, \mathrm{H}$. et al. Morphable 3D mesostructures and microelectronic devices by multistable buckling mechanics. Nat Mater, 1 (2018).

64 Luo, G. Q. et al. Mechanics of bistable cross-shaped structures through loading-path controlled 3D assembly. Journal of the Mechanics and Physics of Solids 129, 261-277, doi:10.1016/j.jmps.2019.05.007 (2019).

65 Seung, H. \& Nelson, D. R. Defects in flexible membranes with crystalline order. Physical Review A 38, 1005 (1988).

66 Grinspun, E., Hirani, A. N., Desbrun, M. \& Schröder, P. in Proceedings of the 2003 ACM SIGGRAPH/Eurographics symposium on Computer animation. $62-67$ (Citeseer).

67 Weinan, E., Ren, W. \& Vanden-Eijnden, E. Simplified and improved string method for computing the minimum energy paths in barrier-crossing events. Journal of Chemical Physics 126, 164103 (2007).

68 Kim, Y., Yuk, H., Zhao, R., Chester, S. A. \& Zhao, X. Printing ferromagnetic domains for untethered fast-transforming soft materials. Nature 558, 274-279, doi:10.1038/s41586-018-0185-0 (2018).

69 Camarillo, D. B., Carlson, C. R. \& Salisbury, J. K. Configuration Tracking for Continuum Manipulators With Coupled Tendon Drive. Ieee T Robot 25, 798-808 (2009). 
70 Fusco, S. et al. An Integrated Microrobotic Platform for On-Demand, Targeted Therapeutic Interventions. Advanced Materials 26, 952-957 (2014).

71 Tang, Y. et al. Leveraging elastic instabilities for amplified performance: Spine-inspired high-speed and high-force soft robots. Sci Adv 6, eaaz6912 (2020).

72 Chen, H.-T., Taylor, A. J. \& Yu, N. A review of metasurfaces: physics and applications. Reports on progress in physics 79, 076401 (2016).

73 Liu, F. et al. in 2018 IEEE International Symposium on Circuits and Systems (ISCAS). 1-5 (IEEE).

74 Seung, H. S. \& Nelson, D. R. Defects in flexible membranes with crystalline order. Physical Review A 38, 1005 (1988). 


\section{Figures}

(a)

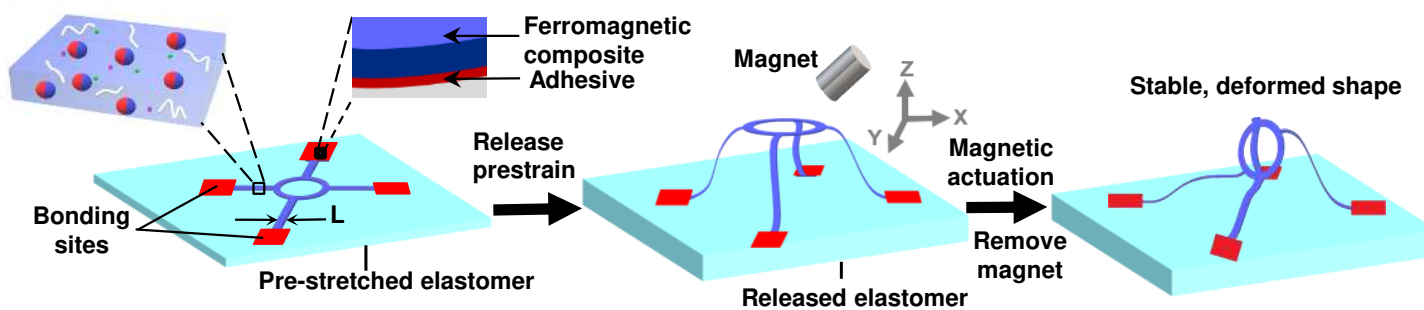

(b)

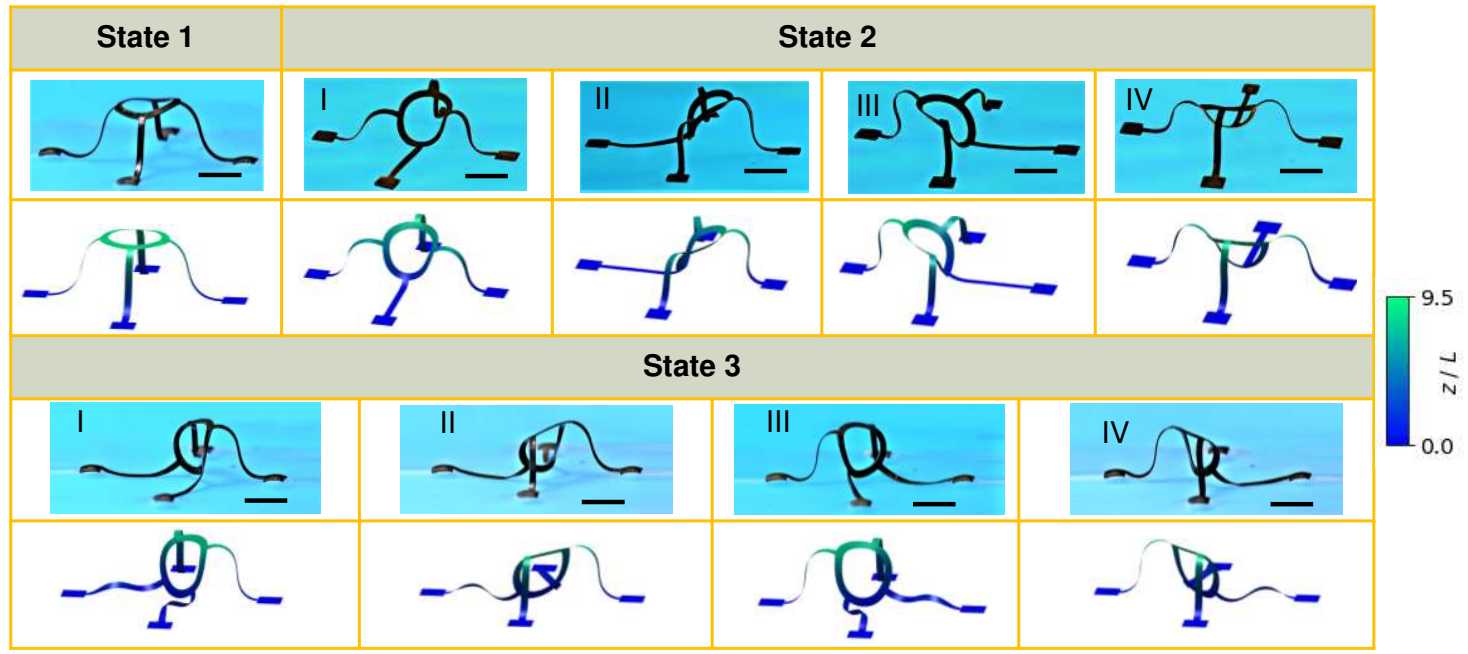

(c)

(d)
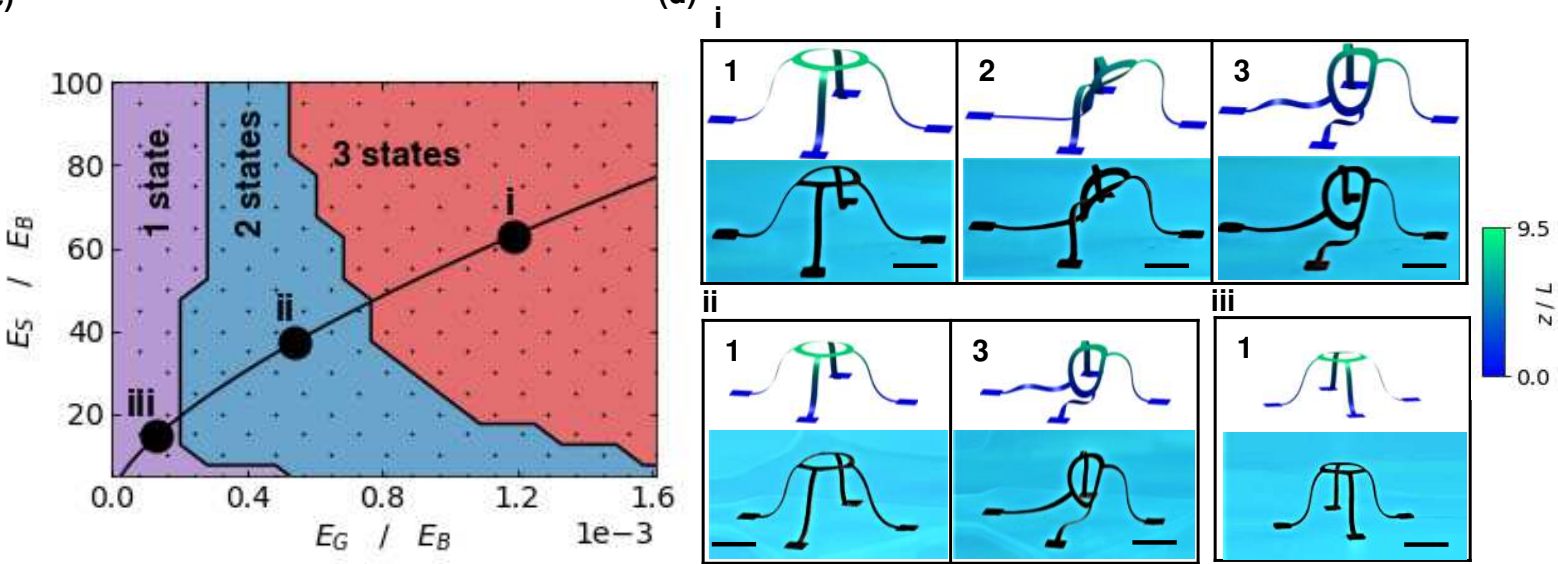

Figure 1. Multistability of magnetically reconfigurable 3D structures. (a) Schematic illustration of the assembly and reconfiguration schemes of 3D ferromagnetic structures. (b) Experimental and computational results of multistable states coexisting in a 3D table structure. Scale bars, $1 \mathrm{~cm}$. (c) Phase diagram showing the number of distinct stable states based on the energy scales of the table structure. The three marked points denote representative examples in each regime. (d) Experimental and computational results of the distinct stable states existing in three table structures of different in-plane dimensions $(L)$, corresponding to the marked points in (c). Scale bars, $1 \mathrm{~cm}$. 
(a)

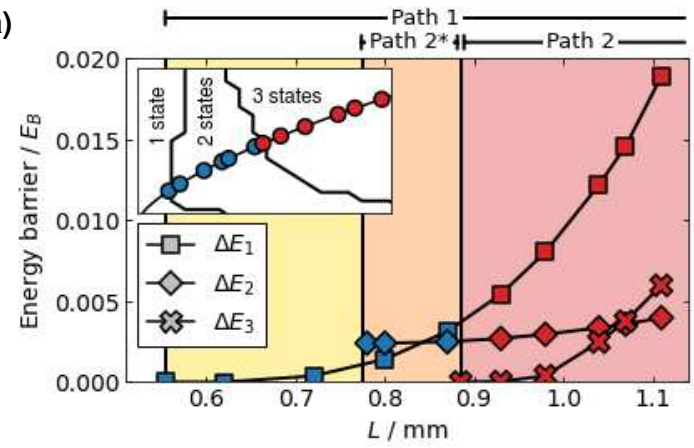

(b) _- Path 1 -- Path 2 … Path 2*

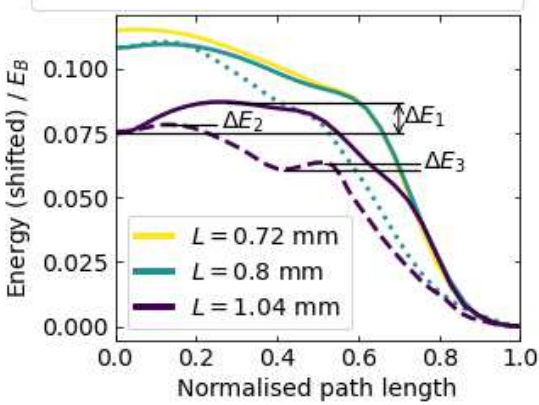

(c) L=1.04 mm, paths 1 and 2 exist

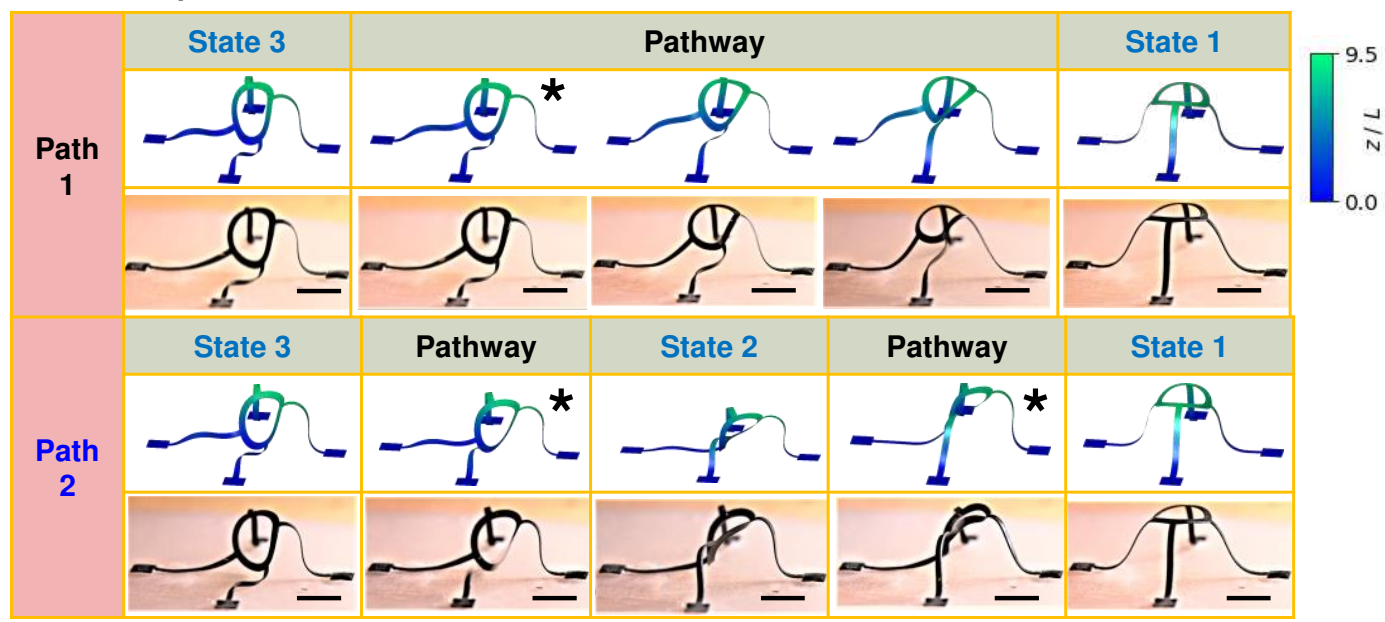

(d) $L=0.8 \mathrm{~mm}$, paths 1 and $2^{*}$ exist

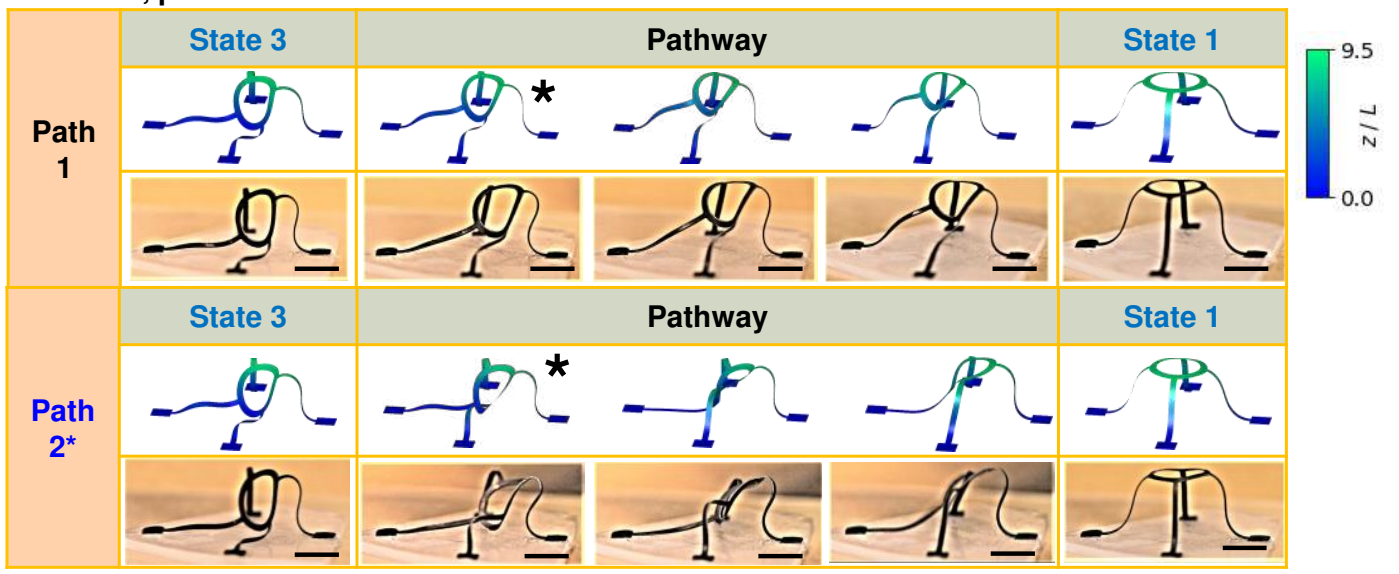

(e) $L=0.72 \mathrm{~mm}$, only path 1 exists

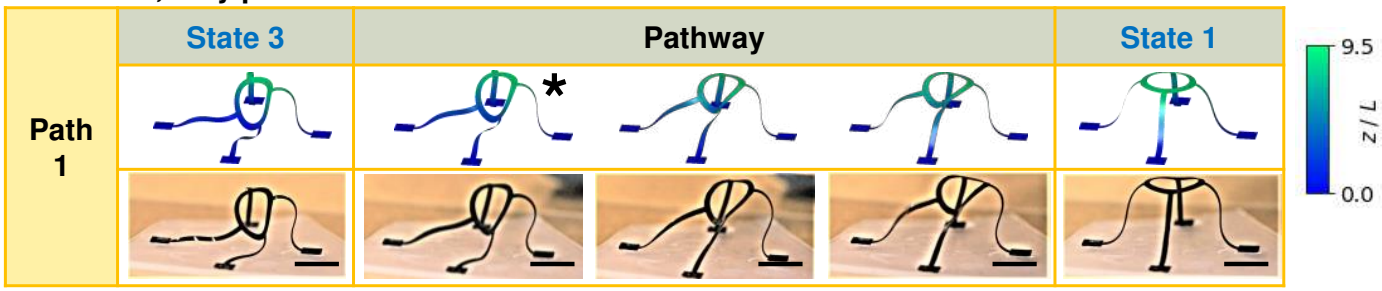

Figure 2. Reconfiguration pathways and energy barriers. (a) Variation of the energy barriers as a function of the in-plane dimension of the table structure, $L$. Three regions with different available pathways from state 3 to 1 of the table structure are highlighted. Inset: location of the marked data points within the phase diagram in Fig. 1c. (b) Energy profiles for pathways of representative cases in each region, with $L=1.04 \mathrm{~mm}, 0.8 \mathrm{~mm}$, and $0.72 \mathrm{~mm}$, respectively. The energies are shifted relative to that of state 1 and scaled by the bending energy scale, with three energy barriers $\left(\Delta E_{1}, \Delta E_{2}, \Delta E_{3}\right)$ labelled. (c-e) Snapshots of configurations along the pathways in (b), with the transition states along the pathways labelled with asterisks. Scale bars, $1 \mathrm{~cm}$. 
(a)

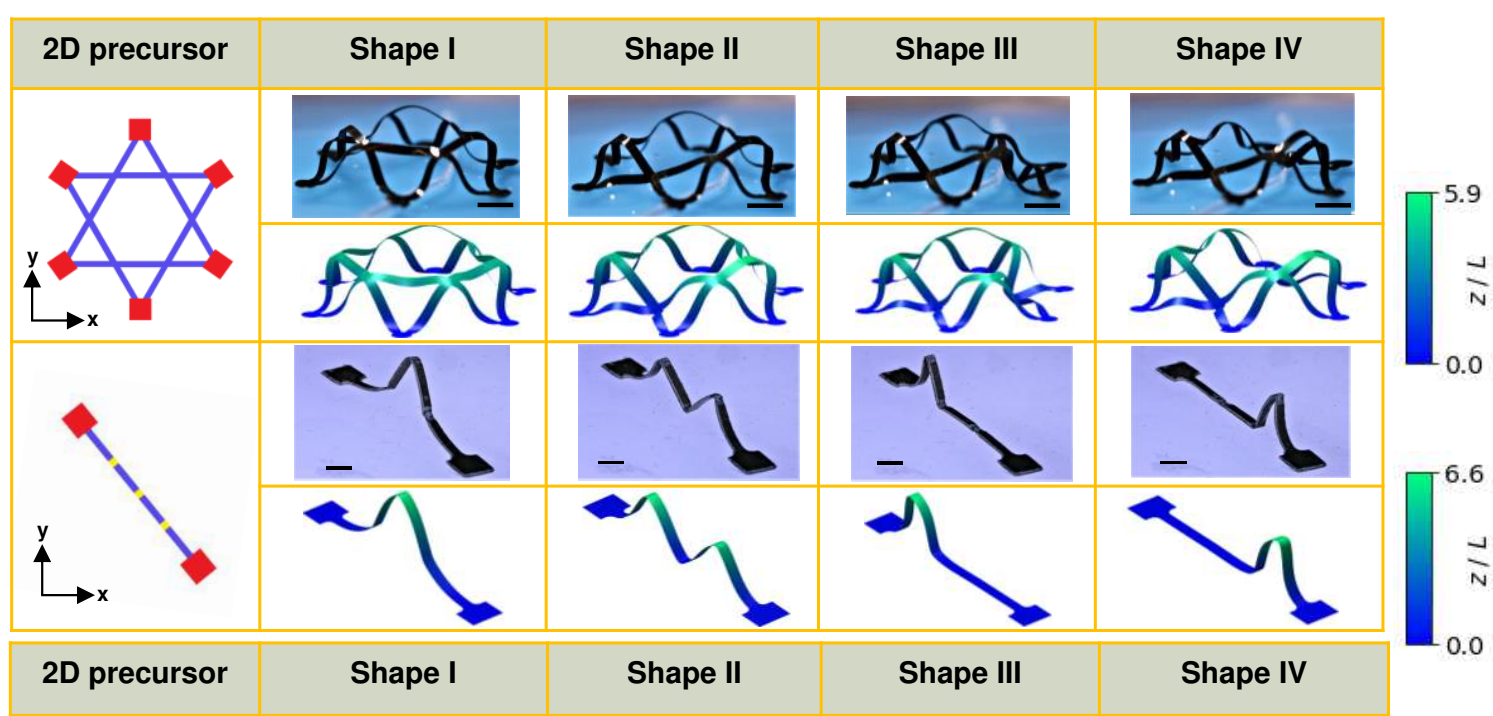

(b)
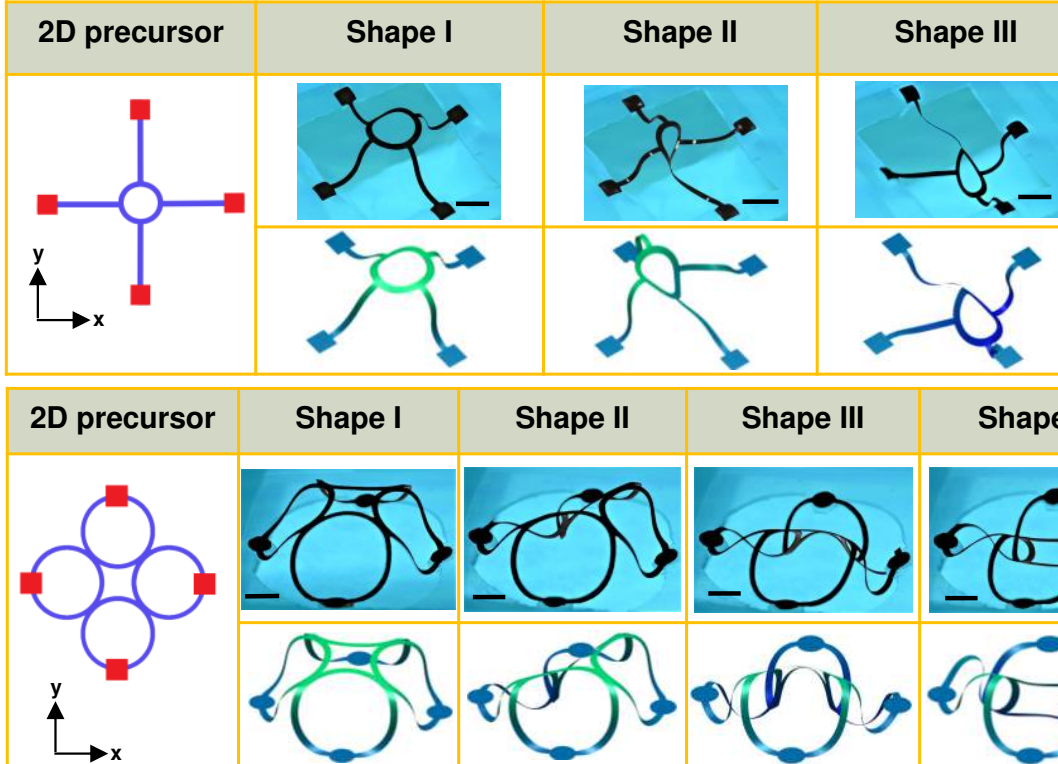

\section{Shape I}

Shape II

Shape III

Shape IV

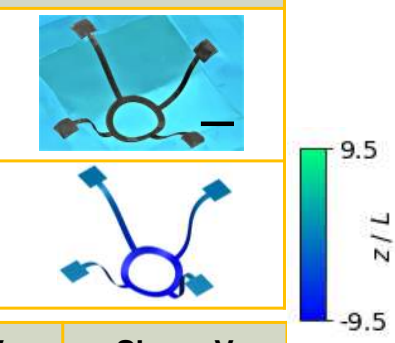

(c)

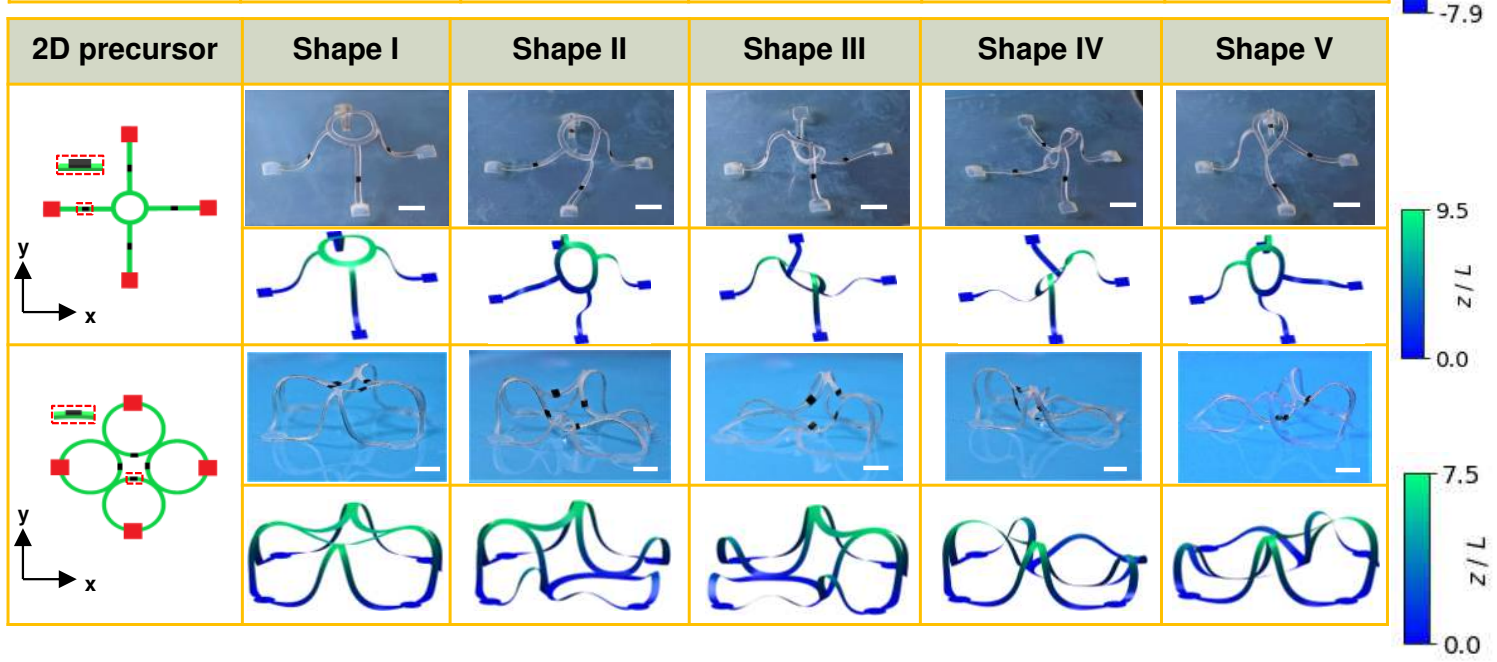

Figure 3. Diverse magnetically reconfigurable 3D structures. Optical images and computational modeling of multistable states for three types of structures: (a) 3D structures attached to a substrate. Scale bars in the first and second structure are $1 \mathrm{~cm}$ and $2 \mathrm{~mm}$, respectively. (b) 3D structures with the substrate underneath the structure removed to allow more freedom for reconfiguration. Please note that the structures are placed vertically, with the direction of gravity along the $x$ direction. Scale bars, $1 \mathrm{~cm}$. (c) 3D structures composed of locally patterned ferromagnetic films on a thin layer of PDMS. Scale bars, $1 \mathrm{~cm}$. 
(a)

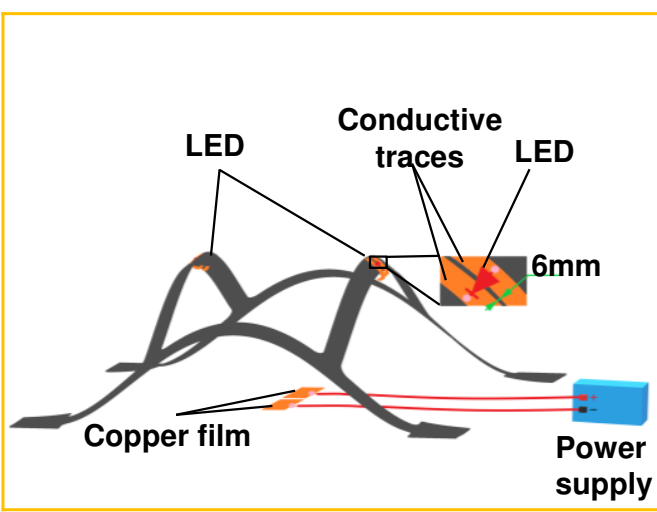

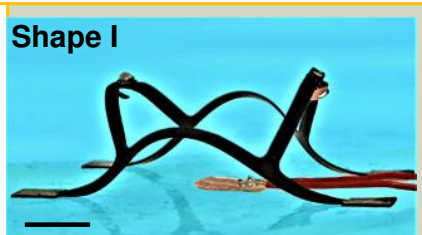

Shape II

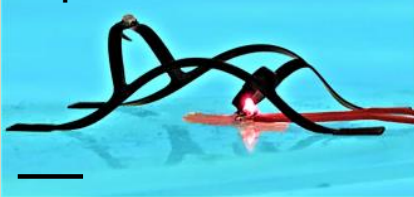

Shape III

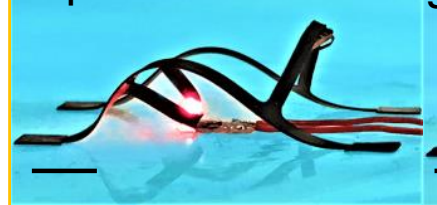

Shape IV

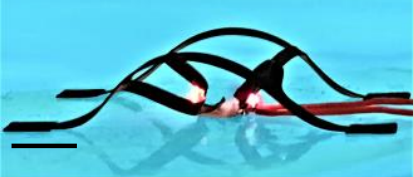

(b) Magnetically Controlled release of particles
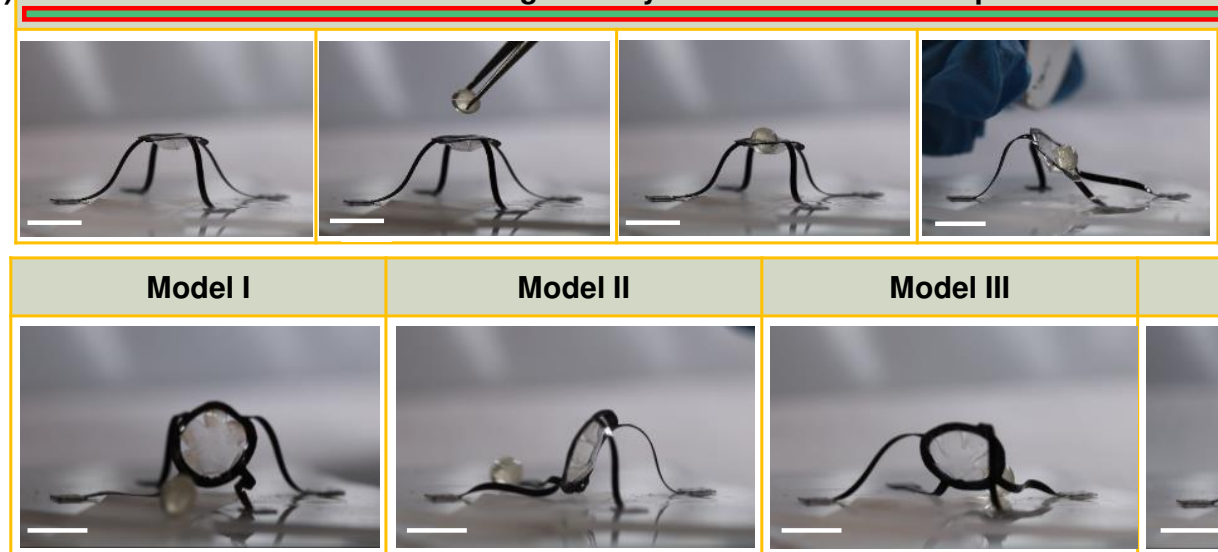

Model II

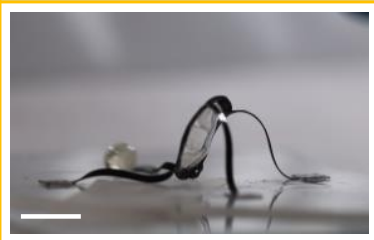

Model III

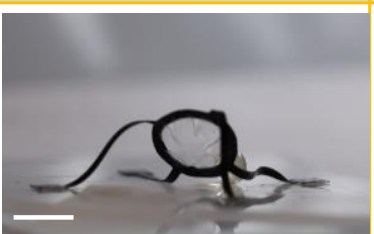

Model IV

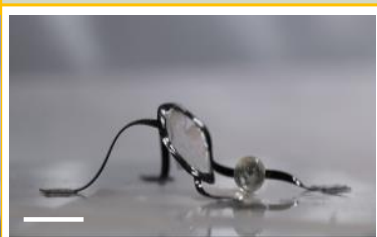

Figure 4. Applications of reconfigurable structures in on-demand 3D light emitting systems and controlled particle delivery. (a) Schematic illustration and optical images of a 3D light emitting system that can be reconfigured among its multistable states to switch the LEDs on and off. Scale bars, $1 \mathrm{~cm}$. (b) Controlled release of particles in four different directions (Modes I-IV) enabled by four possible stable states of the table structure. Scale bars, $1 \mathrm{~cm}$. 
(a)

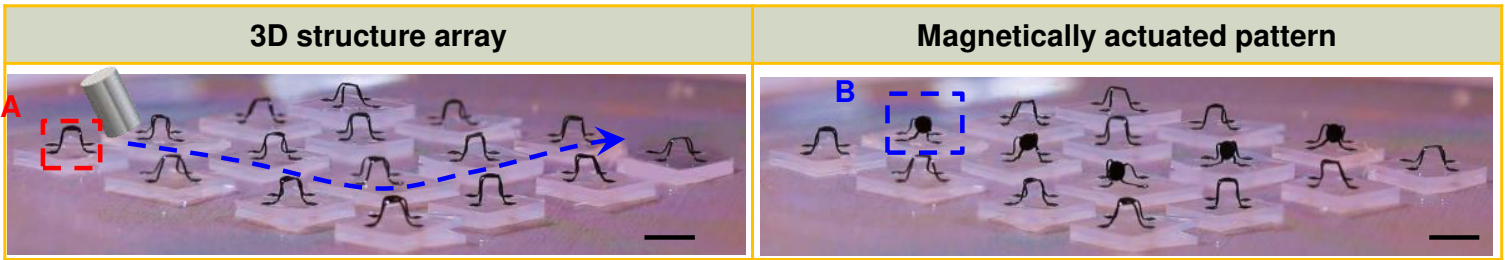

A
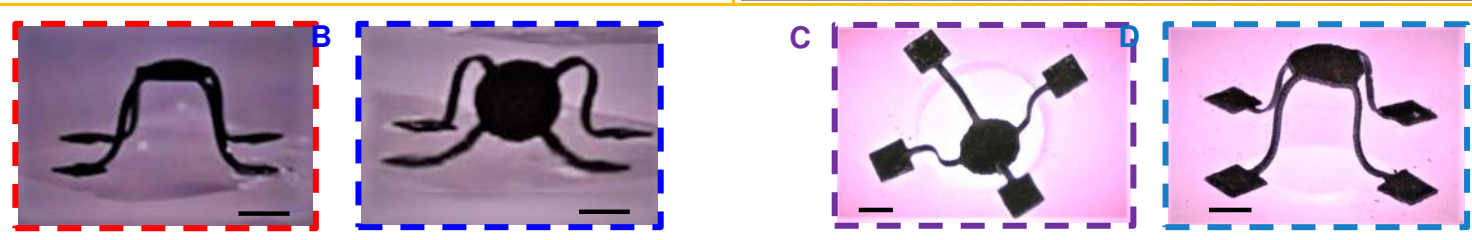

(b)

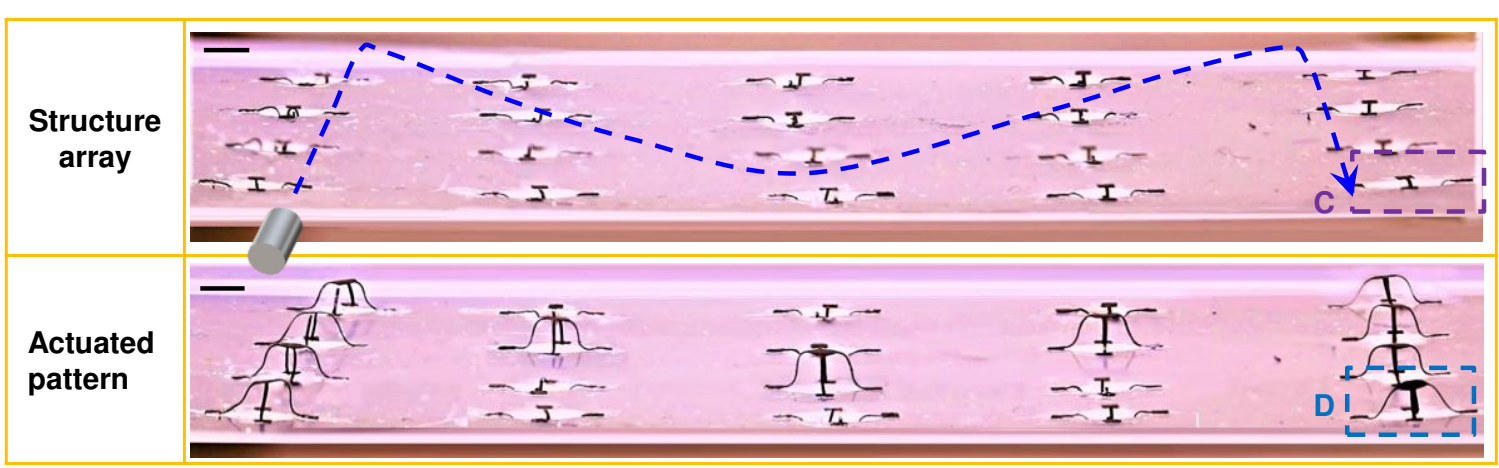

Figure 5. Reconfigurable 3D structure array. (a) A 4×4 array of multistable table structures (on an intact substrate) magnetically deformed to display an alphabetic pattern of "V". Scale bars, $5 \mathrm{~mm}$. Image A-D are magnified views of the 3D structures; Scale bars, $1 \mathrm{~mm}$. (b) A $4 \times 5$ array of multistable table structures (with the substrate underneath the 3D structure removed) magnetically deformed to display an alphabetic pattern of "M". Scale bars, $3 \mathrm{~mm}$. 


\section{Figures}

(a)
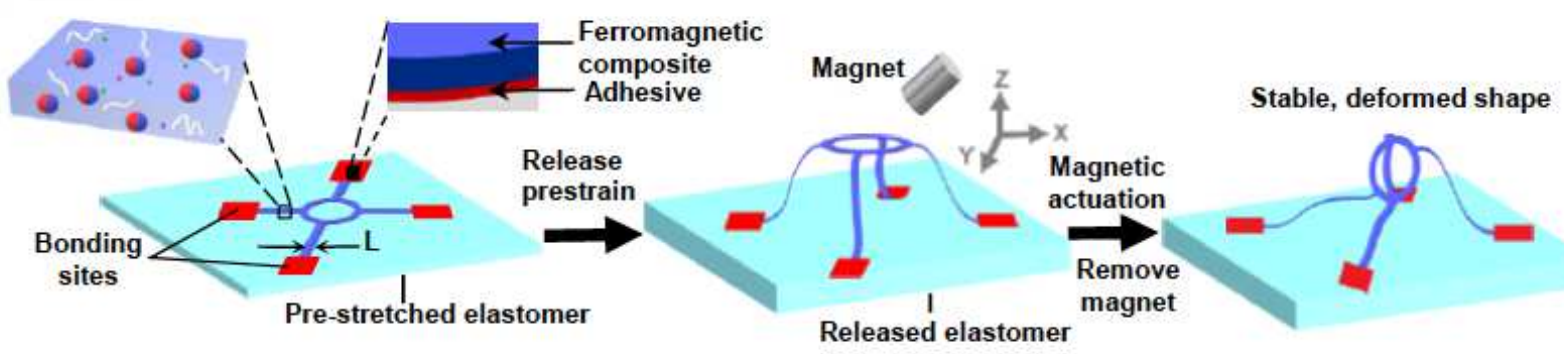

(b)

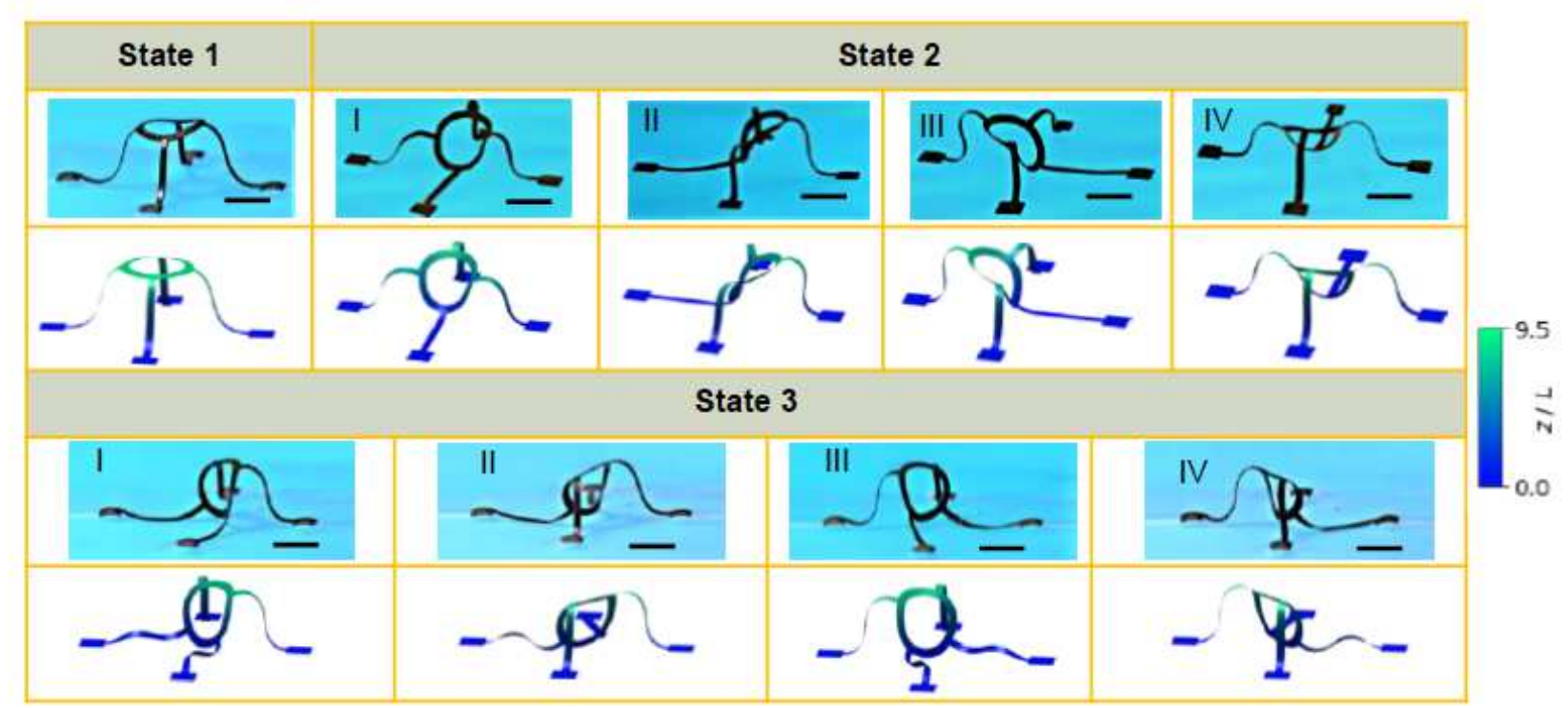

(c)

(d)
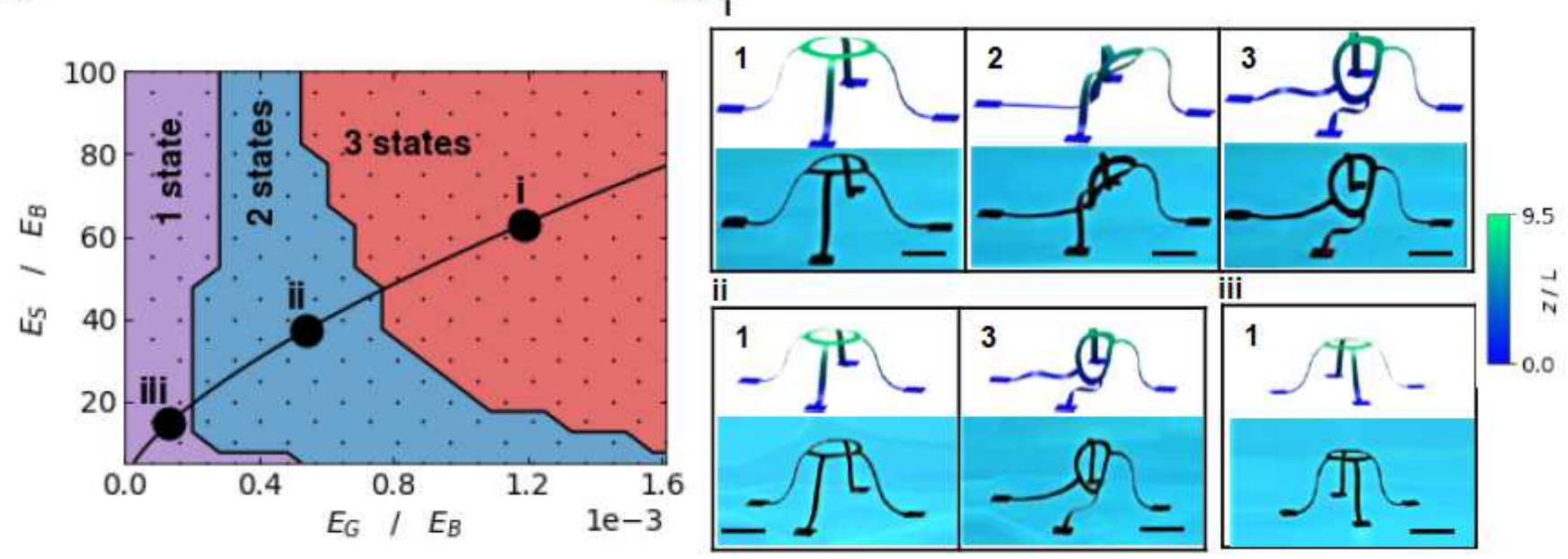

Figure 1

Multistability of magnetically reconfigurable 3D structures. (a) Schematic illustration of the assembly and reconfiguration schemes of 3D ferromagnetic structures. (b) Experimental and computational results of multistable states coexisting in a 3D table structure. Scale bars, $1 \mathrm{~cm}$. (c) Phase diagram showing the number of distinct stable states based on the energy scales of the table structure. The three marked points denote representative examples in each regime. (d) Experimental and computational results of the 
distinct stable states existing in three table structures of different in-plane dimensions $(\mathrm{L})$, corresponding to the marked points in (c). Scale bars, $1 \mathrm{~cm}$.

(a)

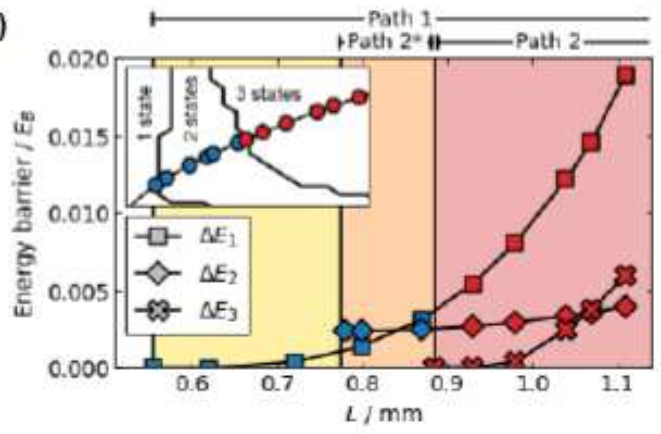

(b) - Path 1 - Path 2 .... Path 2*

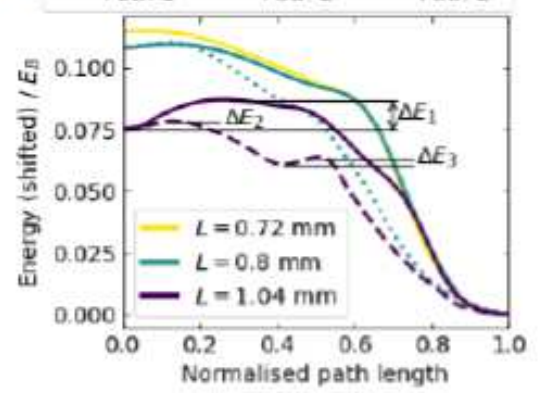

(c) L=1.04 mm, paths 1 and 2 exist

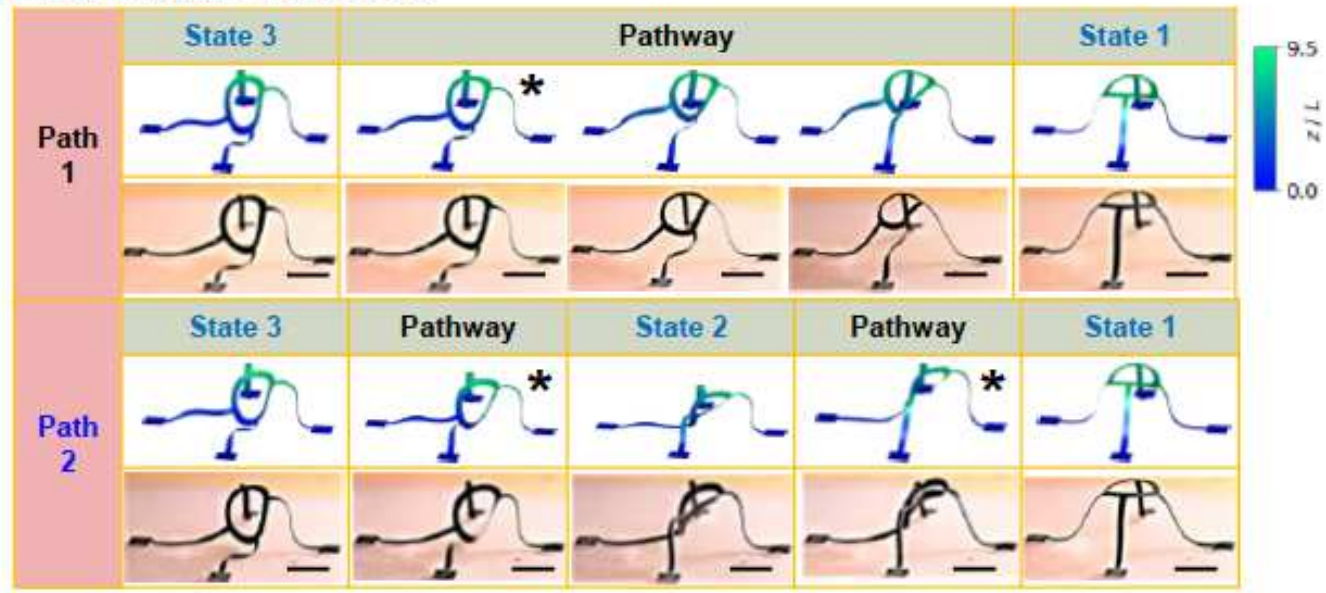

(d) $\mathrm{L}=0.8 \mathrm{~mm}$, paths 1 and $2^{*}$ exist

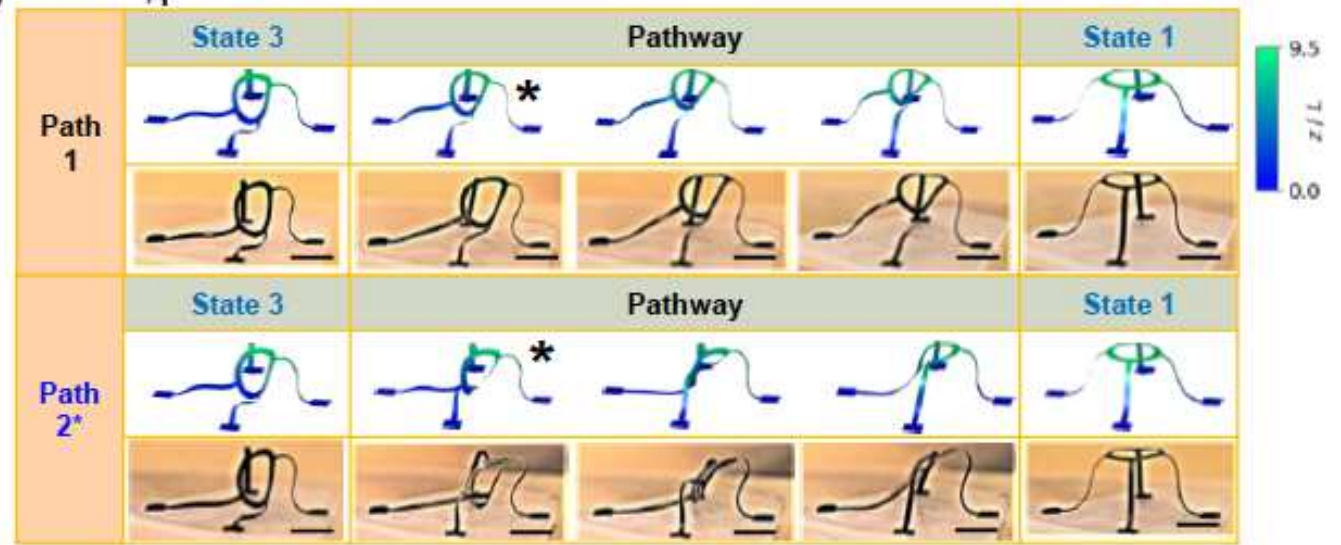

(e) $\mathrm{L}=0.72 \mathrm{~mm}$, only path 1 exists

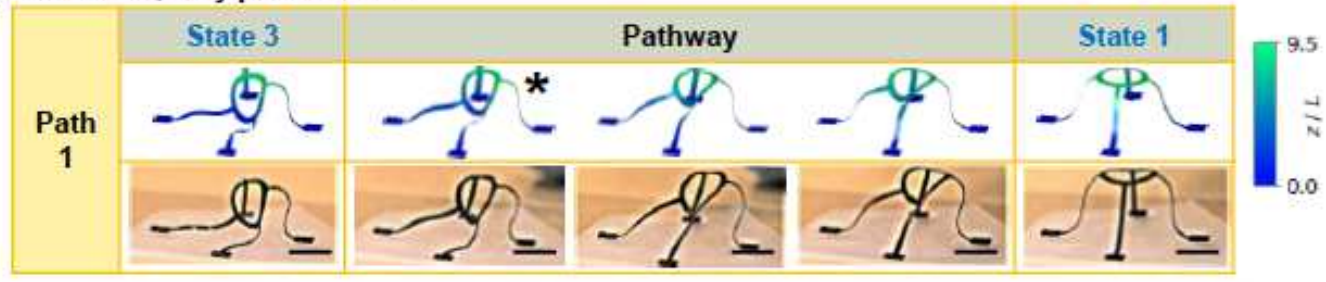

Figure 2

Reconfiguration pathways and energy barriers. (a) Variation of the energy barriers as a function of the inplane dimension of the table structure, L. Three regions with different available pathways from state 3 to 1 of the table structure are highlighted. Inset: location of the marked data points within the phase 
diagram in Fig. 1c. (b) Energy profiles for pathways of representative cases in each region, with $L=1.04$ $\mathrm{mm}, 0.8 \mathrm{~mm}$, and $0.72 \mathrm{~mm}$, respectively. The energies are shifted relative to that of state 1 and scaled by the bending energy scale, with three energy barriers $(\Delta \mathrm{E} 1, \Delta \mathrm{E} 2, \Delta \mathrm{E} 3)$ labelled. (c-e) Snapshots of configurations along the pathways in (b), with the transition states along the pathways labelled with asterisks. Scale bars, $1 \mathrm{~cm}$.

(a)

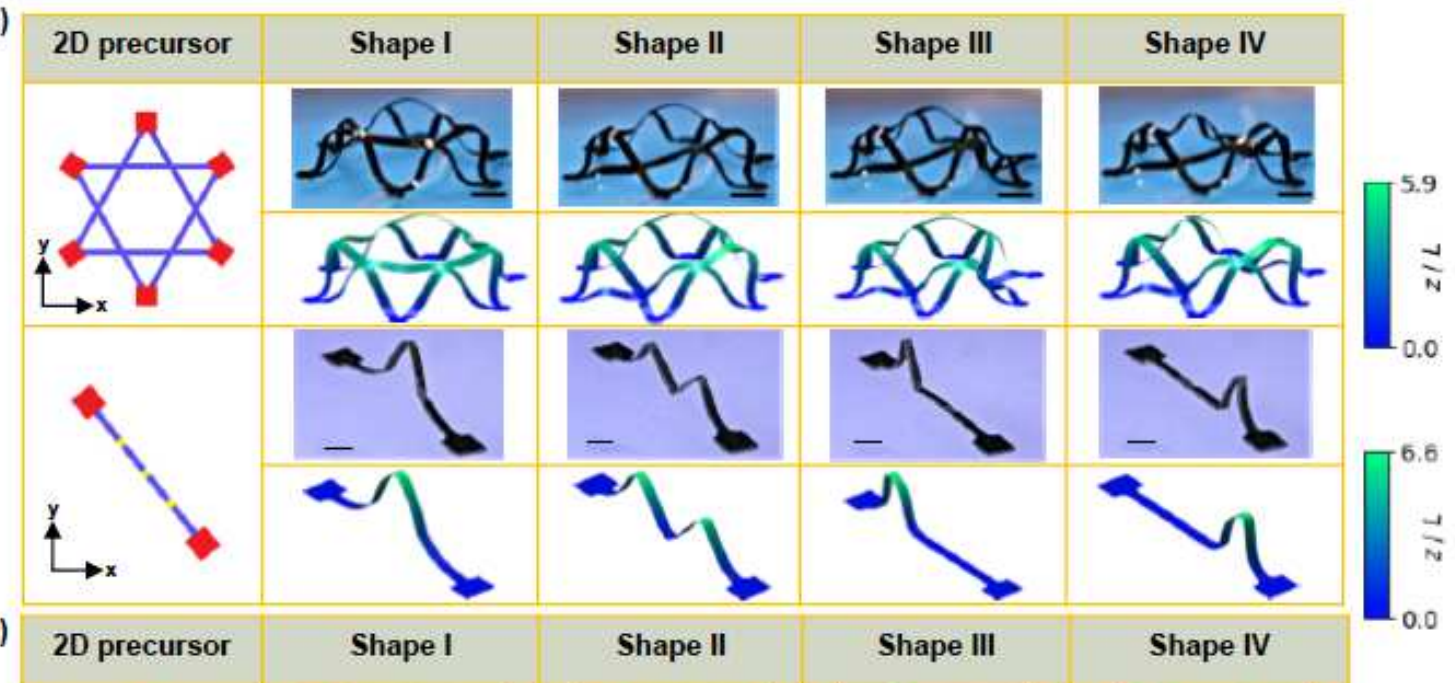

(b)
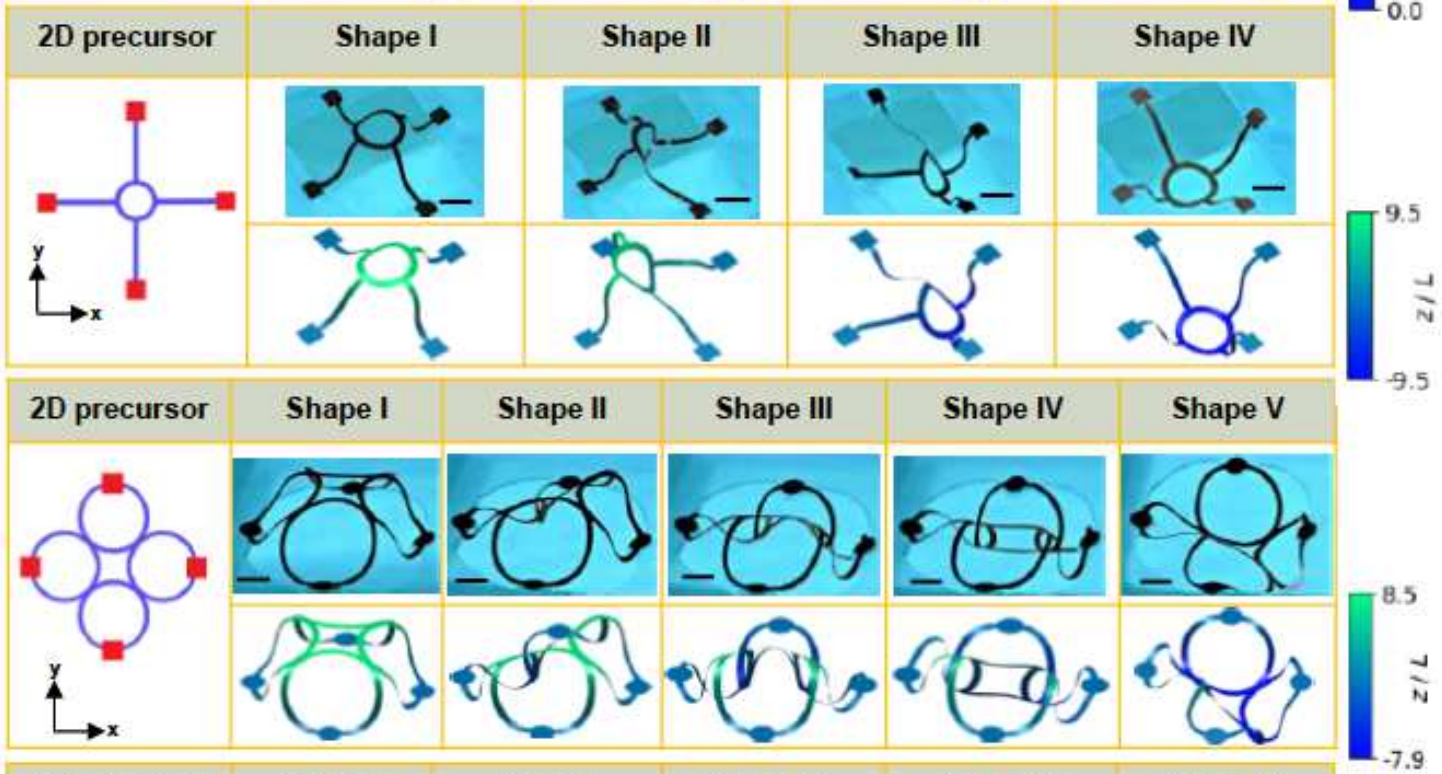

(c)

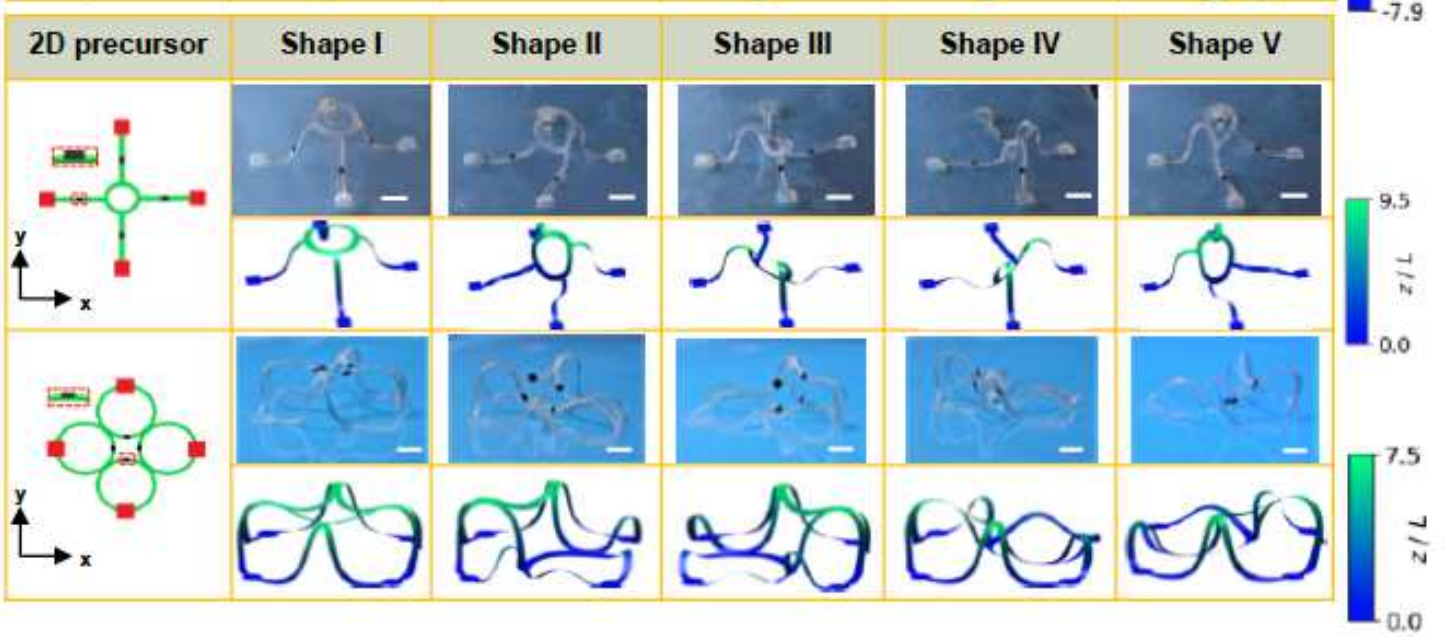

Figure 3 
Diverse magnetically reconfigurable 3D structures. Optical images and computational modeling of multistable states for three types of structures: (a) 3D structures attached to a substrate. Scale bars in the first and second structure are $1 \mathrm{~cm}$ and $2 \mathrm{~mm}$, respectively. (b) 3D structures with the substrate underneath the structure removed to allow more freedom for reconfiguration. Please note that the structures are placed vertically, with the direction of gravity along the $x$ direction. Scale bars, $1 \mathrm{~cm}$. (c) 3D structures composed of locally patterned ferromagnetic films on a thin layer of PDMS. Scale bars, $1 \mathrm{~cm}$.

(a)

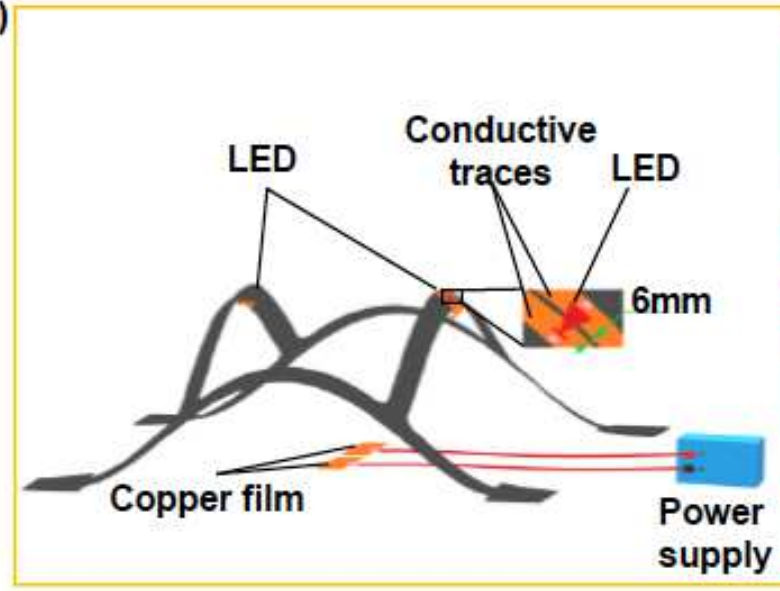

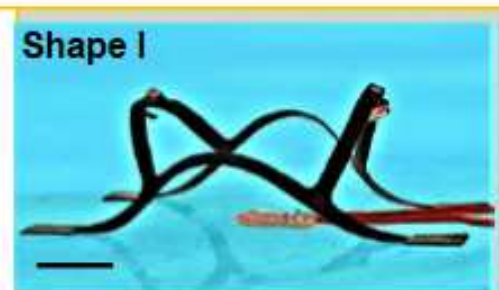

Shape III

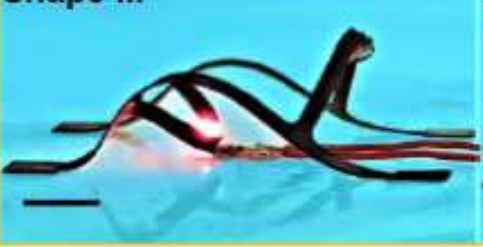

Shape II

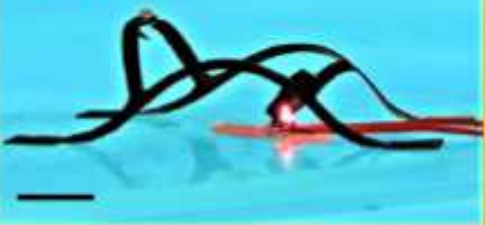

Shape IV

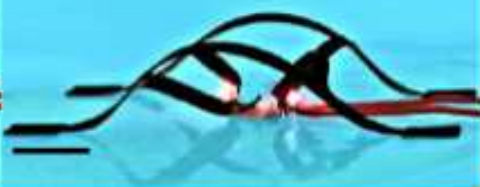

(b) Magnetically Controlled release of particles
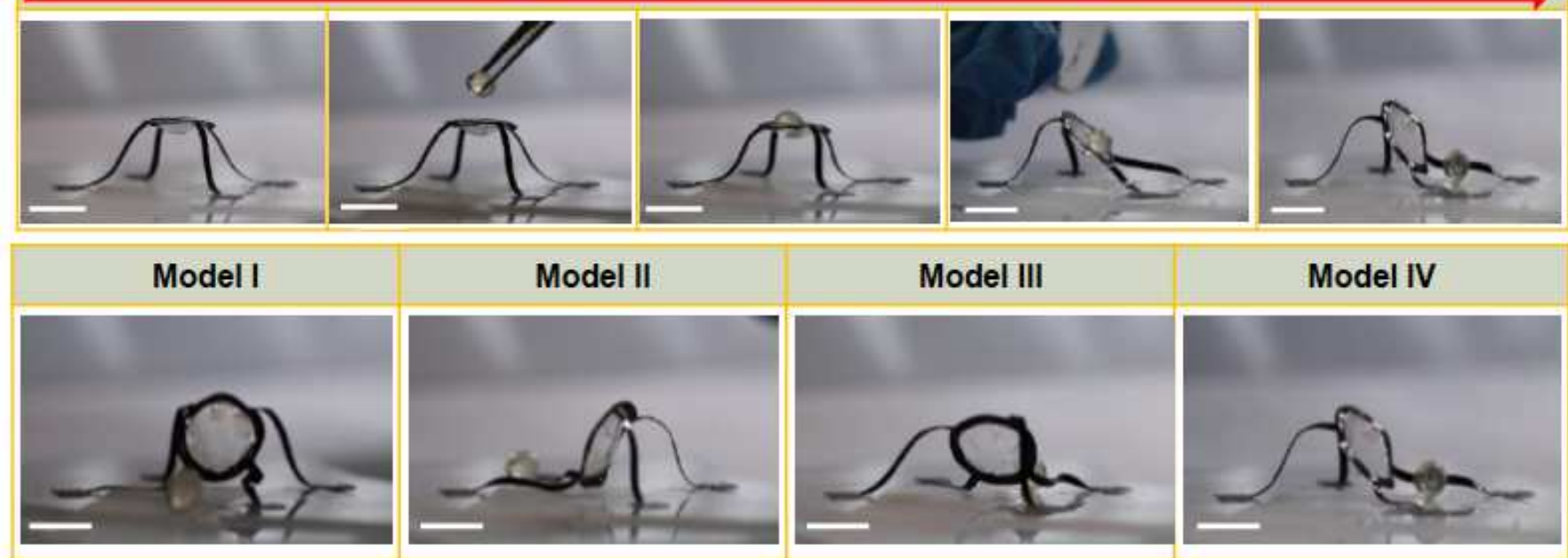

Model II

Model III

Model IV
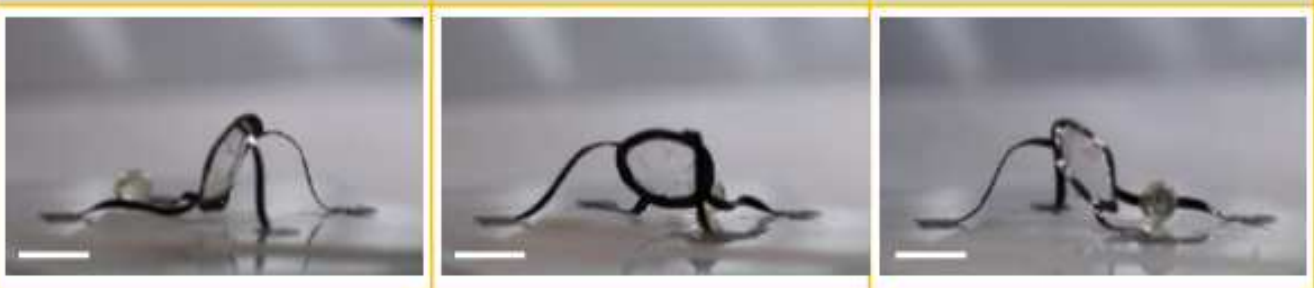

\section{Figure 4}

Applications of reconfigurable structures in on-demand 3D light emitting systems and controlled particle delivery. (a) Schematic illustration and optical images of a 3D light emitting system that can be reconfigured among its multistable states to switch the LEDs on and off. Scale bars, $1 \mathrm{~cm}$. (b) Controlled release of particles in four different directions (Modes I-IV) enabled by four possible stable states of the table structure. Scale bars, $1 \mathrm{~cm}$. 
(a)
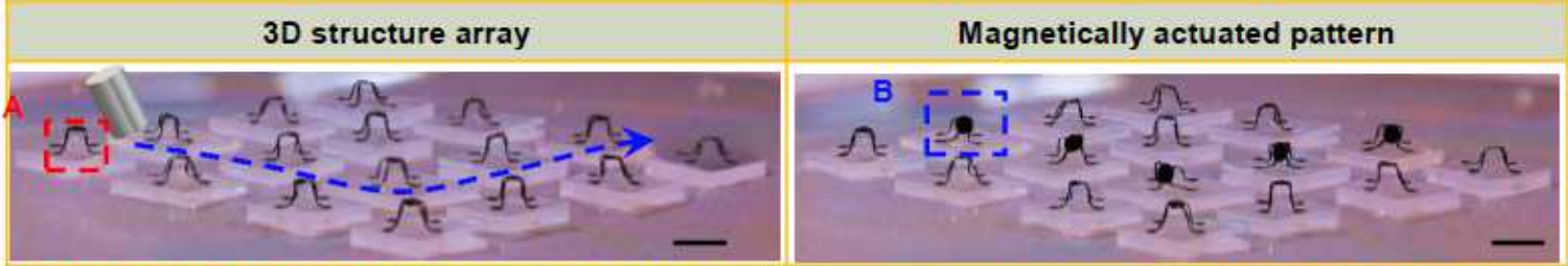

A
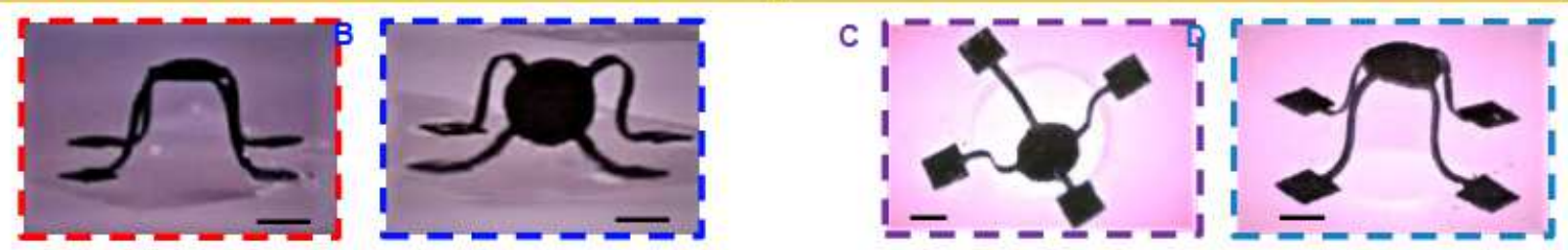

(b)

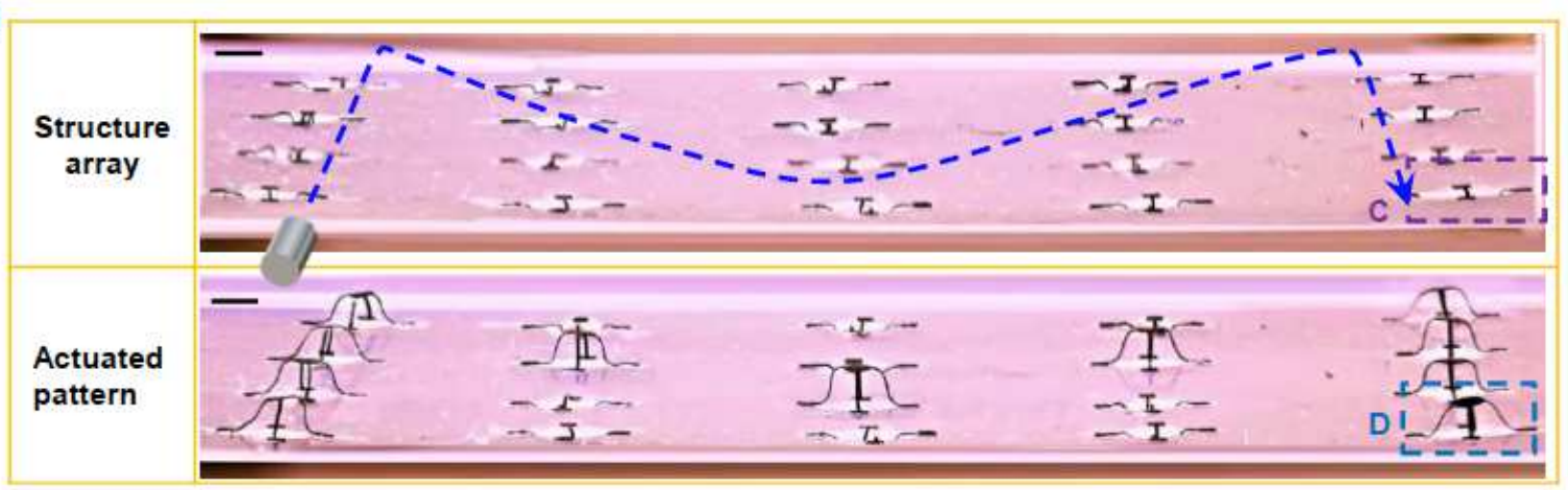

\section{Figure 5}

Reconfigurable 3D structure array. (a) A 4×4 array of multistable table structures (on an intact substrate) magnetically deformed to display an alphabetic pattern of " $V$ ”. Scale bars, $5 \mathrm{~mm}$. Image A-D are magnified views of the 3D structures; Scale bars, $1 \mathrm{~mm}$. (b) A $4 \times 5$ array of multistable table structures (with the substrate underneath the 3D structure removed) magnetically deformed to display an alphabetic pattern of "M". Scale bars, $3 \mathrm{~mm}$.

\section{Supplementary Files}

This is a list of supplementary files associated with this preprint. Click to download.

- SupplementaryMovie1.mp4

- SupplementaryMovie2.mp4

- SupplementaryMovie3.mp4

- SupplementaryMovie4.mp4

- SupplementaryMovie5.mp4

- SupplementaryMovie6.mp4

- SupplementaryMovie7.mp4

- SIreconfigurablestructures0904FINAL.pdf 